\title{
CRITICAL FLUCTUATIONS IN NORMAL-TO-SUPERCONDUCTING TRANSITION
}

\author{
R. Folk, ${ }^{1}$ Yu. Holovatch ${ }^{2}$ \\ ${ }^{1}$ Institut für Theoretische Physik, Johannes Kepler Universität Linz A- \\ 4040 Linz, Austria \\ ${ }^{2}$ Institute for Condensed Matter Physics of the Ukrainian Academy of \\ Sciences, UA-290011 Lviv, Ukraine
}

\section{INTRODUCTION}

Recent advances in our understanding of critical phenomena due to the application ${ }^{1}$ of the renormalization group (RG) approach ${ }^{2}$ are now well known (see e.g. the textbooks $^{3,4,5}$ ). The scale invariance at the critical point and the universality of certain features of critical phenomena can be explained by an RG transformation, and lead to a theory which provides a quantitative description of the critical behaviour of various thermodynamic quantities of interest.

Specifically, the critical point in the RG "language" (in the context of our lecture we specify it as an equilibrium second order phase transition point) corresponds to the stable fixed point of the RG transformation, where the system is scale invariant. Asymptotic properties of the system are governed solely by the coordinate of the stable fixed point whereas non-asymptotic ones are defined in the region of the approach to the fixed point. In addition, the RG approach may permit the order of the phase transition occurring in a certain model to be determined. This is explored by studying the stability of the fixed points of the RG transformation: an absence of a stable fixed point is interpreted as an evidence of a fluctuation-induced first-order phase transition in the system under consideration. For models with no exact solutions or rigorous proofs of the existence of a second order phase transition, that is to say, for the majority of realistic models in statistical physics, RG provides a tool to check the order of the transition.

Now let us define the kind of problem we are going to discuss in this lecture. It can be formulated as: What is the order of the normal-to-superconducting phase transition?. According to the Bardeen-Cooper-Schriefer theory of the superconductivity, the normal-to-superconducting (NS) phase transition is a classical second order phase transition described by the Landau-Ginsburg Hamiltonian with a complex order parameter corresponding to the wave function of the Cooper pairs. Taking into account the fluctuations of the order parameter one can find values of corresponding critical exponents which in this case will coincide with the critical exponents of $O(n)$ symmetric 
field-theoretical model for the case $n=2$ (the XY model). Consequently, this leads to the assertion that the NS phase transition is described by the same set of critical exponents as the phase transition in the normal-to-superfluid liquid. The latter set of exponents has been measured to a high accuracy ${ }^{6}$ and also calculated by various methods $^{7}, 8,9,10,11,12,13,14$.

Taking into account that the corresponding "superfluid liquid" is charged in case of the NS transition, however, complicates the problem. This has been considered first of all by B. I. Halperin, T. C. Lubensky and S. $\mathrm{Ma}^{15}$ and since then different ways of tackling it have been suggested. We shall discuss some of them briefly in the subsequent section.

From an experimental viewpoint, when the question of the order of the superconducting phase transition has been first discussed it has been considered more or less academic since, due to the large correlation length $\xi_{0} \sim 10^{3} \AA$, the first order characteristics can be seen only very near the phase transition; otherwise mean field behaviour is to be expected. This situation has changed after the discovery of high- $\mathrm{T}_{c}$ superconductors with correlation lengths of the order of lattice distances $\left(\xi_{0} \sim 1 \AA\right)^{16}$. Since then, critical effects have been observed in several experiments $17,18,19,20,21,22,23,24$.

Our main result presented in this lecture is that within the framework of the $R G$ method applied to the original superconductor model, minimally coupled to the gauge field ${ }^{15}$, one still can demonstrate the existence of a second order phase transition with critical exponents distinct from those of a superfluid liquid. To prove this we shall consider the two-loop renormalization group functions for the model, paying particular attention to the fact that the loop expansion is asymptotic ${ }^{27,28,29}$. In this way we find several fixed points with new scaling exponents and a rich crossover behavior. Some of our results have been previously published in ${ }^{30,31,32}$.

The lecture is, therefore, organized as follows. In the following section we shall give a brief review of the methods used to study the problem we are interested in. Then we describe the model of a superconductor, provide results of its study by the mean field approximation, obtain the expressions for the renormalization group functions in a two loop approximation and describe the results obtained subsequently without applying any resummation procedure. Then in the next section we shall discuss several well-established examples in the modern theory of critical phenomena where the resummation of the asymptotic series is applied. After that we shall present the pivotal section of our investigation: it is devoted to a study of the RG functions and of the corresponding flows on the basis of the resummation technique. We obtain as a result that a stable fixed point is present, which is an evidence of a second order phase transition in the model. In the remainder of the paper the asymptotic and effective values for the critical exponents are calculated and we give expressions for the amplitude ratios. To conclude, these results are discussed in the closing section.

\section{NORMAL-TO-SUPERCONDUCTING TRANSITION: 1st OR 2nd OR- DER?}

The order of a phase transition may have severe effects on the physical quantities of a material. This is illustrated by the first-order liquid-gas transition phenomena like overheating and undercooling connected with the metastability at the transition. For second order phase transitions, divergences in physical quantities occur (in the thermodynamic limit, of course) leading to a dramatic increase of the specific heat or the scattering of the light (the critical opalescence) near the liquid-gas critical point. Similar dramatic changes are associated with the phase transitions which have occurred in the 
early stages of the universe where the questions discussed here for superconductors also find relevance (for a recent review see $^{33}$ ).

As mentioned in the Introduction, the question of the NS phase transition order becomes complicated when one accounts for the fluctuations of the order parameter being coupled to the "gauge field" (the vector potential of the fluctuating magnetic field created by the Cooper pairs), the fluctuations of which also diverge at long distances. Posed for the first time more than 20 years ago ${ }^{15}$, this problem has remained a challenging one in the physics of the superconductivity until now.

The theoretical model of Halperin, Lubensky, and Ma describing the relevant critical behaviour is the usual $O(n)$ symmetric $\phi^{4}$ model with the $n / 2$-component complex field $\phi$ coupled to a gauge field which describes the fluctuating magnetic field created by Cooper pairs. The answer obtained in ${ }^{15}$ states that due to the coupling to the gauge field, in the mean field approximation a third order term appears in the free energy of the superconductor and the NS phase transition is of first order. This mean field analysis is appropriate for the type-I superconductors ${ }^{34}$ where the fluctuations in the order parameter have no significant effect on the thermodynamics of the transition.

The case of type-II superconductors is more complicated, however, because fluctuations cannot be neglected. Studying the problem within Wilson-Fisher recursion relations $^{35}$ in the first order of $\varepsilon$ it has been found ${ }^{15}$ that a stable fixed point (necessary, but not sufficient for a second order phase transition) exists only for the order parameter components number $n>365.9$, far exceeding the superconductor case $n=2$. The crossover near the first order phase transition has been examined ${ }^{26}$ and the expression for the crossover function of the specific heat is given by the one loop order perturbation theory.

The kinetics of fluctuations arising from vortex pairs in a superconductor has been studied by means of numerical simulations ${ }^{36}$. The result leads to the conclusion that there exist nucleation processes typical for the first order phase transition, confirming the mean field and RG results of ${ }^{15}$. Note, though, that the mean field analysis applied to the Ginzburg-Landau free energy of a superconductor ${ }^{37}$ including a Chern-Simons term leads to quantitatively different behaviour: for different values of the topological mass, there occurs in a system either a fluctuation-induced first order phase transition or only a second-order transition. This result is also confirmed in the framework of one-loop RG calculations ${ }^{37}$.

The occurrence of a first order phase transition has been also found in the massless scalar electrodynamics ${ }^{38,39}$ and confirmed to a linear order in $\varepsilon$ for the $n$-component Abelian Higgs models by an explicit construction of the coexistence curve and the equation of state $^{40}$. In addition, the $\mathrm{CP}^{N-1}$ non-linear sigma model, being related to the superconductor model in the limit of infinite charge by means of the $(2+\varepsilon)$ expansion, has been shown ${ }^{41}$ to posses a similar behaviour observed further by ${ }^{15}$.

Results for type-II superconductors obtained by $\varepsilon$-expansion methods ${ }^{15,26,40}$ appear to be stable against the influence of different physical factors such as the possibility of another (non-magnetic) ordering, the presence of a disorder and a crystal anisotropy when the study is performed by means of a strict expansion. The scaling behaviour of a superconducting system with an additional non-magnetic ordering studied by $\varepsilon$ expansion methods provides one more example of a system where a weak first-order phase transition occurs ${ }^{45}$. The analysis of the influence of quenched impurities on the critical behaviour of superconductors when taking account of the magnetic field fluctuations demonstrates ${ }^{46,47}$ the appearance of a new stable fixed point for $1<n<366$. It has been shown ${ }^{47}$, however, that this describes the critical behaviour in the range of space dimensionalities $d_{c}(n)<d<4$ with $d_{c}(2)=3.8$ and results in a first order phase 
transition.

The RG flow for the superconductor model with quenched impurities has been found $^{48}$ to exhibit a stable focus surrounded by an unstable limit cycle. The second order phase transition behaviour is found to show up inside the limit cycle. Introducing random fields with short and long range correlations does not lead to a second order behaviour in the region of $(d, n)$ near $(3,2)^{49}$. Note however, that studies of the influence of quenched and annealed gauge fields on the spontaneous symmetry breaking, performed in terms of Helmholtz free energy ${ }^{42}$ lead to the conclusion that in the first nontrivial or one-loop approximation in the annealed model the spontaneous symmetry breaking occurs through a first order transition for $d=2,3$ whereas the quenched model displays a continuous phase transition. A more complicated account of fluctuations in the annealed model changes the nature of the transition to a continuous one, but the spontaneous symmetry breaking is absent in the model with a quenched disorder ${ }^{42}$. The combined influence of the crystal anisotropy, the magnetic fluctuations, and the quenched randomness on the critical behaviour of unconventional superconductors $^{50}$ studied by means of the RG analysis within the $\varepsilon$-expansion ${ }^{51}$ gives that only fluctuation-induced first order transitions should occur in unconventional superconductors in the vicinity of the critical point.

The results of mean field calculations, however, have been questioned by Lovesey ${ }^{42}$, mentioned above, who has shown that taking into account the gauge field fluctuations in the calculation of the free energy leads back to a second order phase transition. A further indication of a second order phase transition has come several years later when this question has been studied as a lattice problem by means of Monte-Carlo calculations and duality arguments ${ }^{43}$. These results have confirmed that there are scenarios of the NS transition that differ from those obtained in ${ }^{15}$. Namely, the NS transition has been found to be of second order, asymptotically equivalent to that of a superfluid with a reversed temperature axis. Subsequent MC simulations ${ }^{44}$ performed in different regions of couplings lead to the result that the NS transition is strongly first order deep in the type-I region and becomes more weakly first order moving in the direction of the type-II region. Beyond a certain point the data reported in ${ }^{44}$ suggests a second-order transition. The corresponding $O(n)$ nonlinear $\sigma$-model coupled to an Abelian gauge field studied near two dimensions by $2+\varepsilon$ expansion $^{53}$ does not show a first order phase transition either.

The existence of a tricritical point, where the order of the phase transition changes from second into first, has been predicted ${ }^{58}$ by representing the $3 d$ superconductor model by a disordered field theory. The position of the tricritical point is located slightly in the type-I region for the values of the Ginzburg parameter ${ }^{34} k<0.8 / \sqrt{2}$. Starting from the dual formulation of the Landau-Ginzburg theory, by means of the RG arguments, it has been shown that the critical exponents of the NS transition coincide with those of a superfluid transition with a reversed temperature axis ${ }^{59}$. But, while the correlation length critical exponent of the normal-to-superconducting transition is predicted to coincide with the ordinary 3D XY model, the divergency of the renormalized penetration depth is characterised by the mean field value $\nu=1 / 2^{59}$.

The influence of the critical fluctuations on the order of the NS transition has been reconsidered on the basis of the field theoretical RG ideas in ${ }^{30}$. Here the two-loop flow equations ${ }^{30}$ for the static parameters and the $\zeta$-functions ${ }^{52}$ are obtained and it is indicated that a stable fixed point possibly exists and, as a consequence, a second order phase transition may occur. An attractive feature of the flow found $\mathrm{in}^{30}$ is that it discriminates between type-I and type-II superconductors, depending on the initial (background) values of the couplings. For small values of the ratio (coupling to the 
gauge field)/(fourth order coupling) (appropriate for type-II superconductors) the flow comes very near to the fixed point of the uncharged model but ends in a new superconducting fixed point. For large values of the ratio (type-I superconductors) the flow runs away. For intermediate values of the ratio, the critical behavior may be influenced by a second (unstable) superconducting fixed point with scaling exponents quite different from those for the uncharged model.

A flow picture qualitatively similar to ${ }^{30}$ has been obtained $i^{54}$ by solving the model of a charged superconductor approximately with the help of nonperturbative flow equations, a method which appears to give very encouraging results for critical scalar field theories ${ }^{55,56}$. Depending on the relative strength of the ratio (coupling to the gauge field)/(fourth order coupling) a first or a second order phase transition has been found. An approximate description of the tricritical behaviour has been given as well as an estimate of the correlation length critical exponent $\nu$ and the pair correlation function critical exponent $\eta$, which give us the second order phase transition has been reported. Depending on three different assumptions for the stable fixed point value of the coupling to the gauge field, the following values are obtained in two successive truncations of the potential; $(\eta, \nu)=[(-0.13,0.50) ;(-0.20,0.47)],[(-0.13,0.53) ;(-0.17$, $0.58)],[(-0.13,0.59) ;(-0.15,0.62)]$, indicating that the critical exponents belong to the physical region $\eta>2-d$ and $\nu>0$, independent of the truncation, clearly pointing towards a second order phase transition.

In the context of baryogenesis the question of the NS phase transition order has been considered within the two loop approximation in $^{57}$ and the effective potential has been calculated. The $\varepsilon$-expansion has been applied to the electroweak phase transition in order to estimate various parameters of it in leading and next-to-leading orders in $\varepsilon$, including the scalar correlation length, the latent heat, the surface tension, the free energy difference, the bubble nucleation rate, and the baryon nonconservation rate. Of course, the transition has been found to be a first order since only run away flows occur in the strict $\varepsilon$-expansion perturbation theory. Note, that in the electroweak scenario of the baryogenesis there exists a so-called Sakharov requirement which is met when the transition is strongly first order rather than second order.

The NS transition problem has been also studied by an analytical method which is not based on $\varepsilon$ or $1 / n$ expansions. Using a non-perturbative method of solving the approximate Dyson equation for arbitrary $d$ and $n^{60}$ it has been found ${ }^{61}$ that the NS phase transition is governed by a "charged" fixed point. The value of the pair correlation function critical exponent $\eta$ at $d=3, n=2$ is $\eta(3,2)=-0.38$. It is interesting to note that although the result for $\eta$ appears to be a well-behaved function of $d$ and $n$, it breaks down at the critical value $n_{c} \simeq 18$ when expanded in $\varepsilon$. Hence, the conclusion is drawn that the results of the $\varepsilon$-expansion obtained in ${ }^{15}$ and, in particular, the absence of a stable fixed point solution for $n<n_{c} \simeq 365.9$ are to be interpreted as the breakdown of the $\varepsilon$-expansion rather than a fluctuation-induced first-order phase transition. On the other hand, near $d=4$ the results of ${ }^{61}$ are in a good agreement with the $\varepsilon$-expansion data $^{15}$ for high $n(n>366)$.

Recently, the same problem studied with the RG technique in a fixed dimension $d=3$ within the one-loop approximation has given an evidence of an attractive charged fixed point distinct from that of a neutral superfluid, leading, in particular, to the correlation length critical exponents values $\nu \simeq 0.53$ and $\eta \simeq-0.70{ }^{62}$. Considered in the form of the continuum dual theory ${ }^{63}$, however, the magnetic penetration depth has been shown to diverge with the XY exponent, contradicting results mentioned above $^{30,54,61,62}$. To investigate this discrepancy, MC simulations of the 3D isotropic lattice superconductor in a zero external magnetic field have been performed. This 
results in the conclusion that there is a single diverging length scale consistent with the universality of the ordinary 3D XY model ${ }^{65}$. Further applications of the model containing coupling to the gauge field have been suggested in the context of the quantum Hall effect ${ }^{66}$.

Now, let us give a brief resume of the experimental data relevant to our study. As mentioned in the Introduction, the effects of thermodynamic fluctuations are generally small in conventional low- $T_{c}$ superconductors because of their low transition temperatures and large coherence length. In contrast, high transition temperatures and small coherence lengths mean that critical fluctuations are relevant in high- $T_{c}$ superconductors. Though critical fluctuations in high- $T_{c}$ superconductors have been observed in a series of experiments ( see e.g. ${ }^{17}, 18,19,20,21,22,23,24$ ) their interpretation has been changed somewhat. Deviations from the mean field (i.e. first order) behaviour have accounted for either by $3 d$ Gaussian fluctuations (giving, in particular, values for the specific heat critical exponent $\alpha$ and the correlation length critical exponent $\nu$ : $\alpha=\nu=1 / 2)^{17,19}$ or by a nontrivial XY behaviour characteristic for an uncharged superfluid (with $\nu \simeq 2 / 3$ and logarithmic divergences in $\alpha)^{18,20,22,24}$. Measurements of the heat capacity ${ }^{22,24}$, the magnetization and the electric conductivity ${ }^{22}$ of singlecrystal samples of $Y \mathrm{Ba}_{2} \mathrm{Cu}_{3} \mathrm{O}_{7-x}$ in a magnetic fields near $T_{c}$ support the existence

of a critical regime governed by the XY-like critical exponents ${ }^{18,20,22,24,25}$, a similar conclusion follows from the crossover analysis of the zero-field heat capacity on a comparable sample ${ }^{21}$. The maximum applied magnetic field for which the 3D XY scaling is valid, however, differs for various materials ${ }^{67}$.

To conclude this brief review it is worth mentioning one more physical interpretation of a charged field coupled to the gauge vector potential. Namely, this is the nematic-smectic-A transition in liquid crystals $68,69,70,71,72,73$. The nematic phase is an orientationally ordered but translationally disordered phase, rodlike molecules are aligned with their long axes parallel to the director and the smectic-A phase contains layers of molecules with their long axes perpendicular to the layer. It has been proposed $^{68,69}$ that this transition can be described by a model similar to those describing the NS transition in the charged case $^{15}$. Now the smectic order parameter (being a complex field $\Psi(r)$ that specifies the amplitude and the phase of the density modulation induced by the layering) is coupled to the director fluctuations. Contrary to the NS transition, the nematic-smectic A transition is characterized by a critical region in the experimentally accessible range. For certain materials it has been indeed shown ${ }^{72}$ that both the latent heat data obtained through an adiabatic scanning calorimetry as well as independent interface velocity measurements near the Landau tricritical point can be fitted by a crossover function consistent with a mean field free energy density that has a cubic term ${ }^{15}$, implying that the nematic-smectic-A transition is a weakly first order. Many liquid crystals, though, appear to exhibit a continuous nematic-smectic-A transition (see ${ }^{73}$ and references therein). High-resolution heat-capacity and x-ray studies of the nematic-smectic-A transition performed during the past twenty years (see ${ }^{73}$ for a comprehensive review) show complex systematic trends to crossover, from threedimensional XY to tricritical behaviour and an anisotropic behaviour due to a coupling between the smectic order parameter and director fluctuations.

\section{THE MODEL AND ITS "NAIVE" ANALYSIS}

Now it is well-known, that the influence of the order parameter fluctuations on the NS transition can be described by the Landau-Ginsburg free energy functional: 


$$
F[\phi]=\int \mathrm{d}^{3} x\left\{\frac{t_{0}}{2}\left|\phi_{0}\right|^{2}+\frac{1}{2} \mid\left(\left.\nabla \phi_{0}\right|^{2}+\frac{u_{0}}{4 !}\left|\phi_{0}\right|^{4}\right\},\right.
$$

$t_{0}$ is temperature-dependent, $u_{0}$ is a coupling constant and the complex order parameter $\phi_{0}$ is connected with the wave function of Cooper pairs. The Cooper pairs are charged and therefore create a fluctuating magnetic field which leads to the appearance of additional terms in the free energy functional. Note, that this is not the case of a normal-to-superfluid transition in a neutral (uncharged) fluid, which is well described by (1) without any modification. Describing the fluctuating magnetic field $\mathbf{B}$ by the vector potential $\mathbf{A}(\mathbf{B}=\operatorname{rot} \mathbf{A})$ and adding to (1) the minimal coupling between the fluctuating vector potential and the order parameter one obtains the free energy functional $F[\Psi, \mathbf{A}]$, originally considered in ${ }^{15}$ for a generalized superconductor in $d$ dimensions with a $d$ dimensional vector potential $\mathbf{A}$ and the order parameter $\Psi$ consisting of $n / 2$ complex components.

One can now describe the fluctuation effects by an Abelian Higgs model with the gauge invariant Hamiltonian ${ }^{15}$ :

$$
H=\int \mathrm{d}^{d} x\left\{\frac{t_{0}}{2}\left|\Psi_{0}\right|^{2}+\frac{1}{2}\left|\left(\nabla-i e_{0} \mathbf{A}_{0}\right) \Psi_{0}\right|^{2}+\frac{u_{0}}{4 !}\left|\Psi_{0}\right|^{4}+\frac{1}{2}\left(\nabla \times \mathbf{A}_{0}\right)^{2}\right\},
$$

which depends on the bare parameters $t_{0}, e_{0}, u_{0}$. The parameter $t_{0}$ changes its sign at some temperature, the rest of the parameters are considered temperature-independent. When the coupling constant $e_{0}=0$ no magnetic fluctuations are induced and the model reduces to the usual field theory (1) describing a second-order phase transition and corresponding in the particular case $n=2$ to the superfluid transition in ${ }^{4} \mathrm{He}$.

The mean field results for the critical behaviour of the model with a free energy functional $F[\Psi, \mathbf{A}]$ corresponding to the Hamiltonian (2) have been already reported in the original paper of Halperin, Lubensky and $\mathrm{Ma}^{15}$. In the framework of the mean field theory one can determine that systems characterized by the free energy functionals $F[\phi]$ (11) and $F[\Psi, \mathbf{A}](n=2)$ possess a qualitatively different critical behaviour. Neglecting the order parameter fluctuations (in accordance with the Ginzburg criterion this may be done for a good type-I superconductor) shows that depending on the sign of $t_{0}$ the free energy (11) is minimized by the value of the order parameter $\phi=0$ for $t_{0}>0$ or by a non-zero value, when $t_{0}<0$, and that the appearance of the non-zero order parameter is continious; in the system under consideration a second order phase transition occurs.

When applied to the free energy functional $F[\Psi, \mathbf{A}]$, however, the mean field theory predicts a qualitatively different behaviour. Defining the effective free energy $F_{\text {eff }}[\Psi]$ as a function of the single variable $\Psi$ by taking the trace over the configurations of the vector potential $\mathbf{A}$ one finds ${ }^{15}$ that the expression for $F[\Psi]$ will contain a term which has a negative sign and is proportional to $|\Psi|^{3}$. Such a term inevitably leads to a first order transition; $F_{\text {eff }}[\Psi]$ develops a minimum at a finite value of $\Psi$ when the coefficient of the quadratic term is still slightly positive.

As it has been already mentioned, the above reasoning is appropriate for a type-I superconductor. The case of type-II superconductors is considerably more complicated. Here, fluctuations in $\Psi$ cannot be neglected and one must choose an appropriate technique to study the problem. Originally, the critical behaviour of the model (2) in the presence of order parameter fluctuations has been studied in ${ }^{15}$ with the help of WilsonFisher recursion relations ${ }^{35}$ in the first order of $\varepsilon=4-d$ and in result it is shown, 
in particular, that the second order phase transition is absent for $n=2$ in the region of couplings appropriate for the type-II superconductor. We will reproduce these $\varepsilon$-expansion results below and then continue to analyze the problem further.

In order to describe the long-distance properties of the model (2) arising in the vicinity of the phase transition point we shall use a field-theoretical RG approach. Twoloop results ${ }^{30}$ for the RG functions corresponding to (2) are obtained on the basis of a dimensional regularization and a minimal subtraction scheme ${ }^{74}$, defining the renormalized quantities so as to subtract all poles at $\varepsilon=4-d=0$ from the renormalized vertex functions. The renormalized fields, mass and couplings are introduced by:

$$
\begin{aligned}
& \Psi_{0}=Z_{\Psi}^{1 / 2} \Psi, \quad \mathbf{A}_{0}=Z_{\mathrm{A}}^{1 / 2} \mathbf{A}, \quad t_{0}-t_{0 c}=Z_{t} Z_{\Psi}^{-1} t, \\
& e_{0}^{2}=Z_{e}^{1} Z_{\mathrm{A}}^{-1} Z_{\Psi}^{-1} e^{2} \mu^{\varepsilon} S_{d}^{-1}, \quad u_{0}=Z_{u} Z_{\Psi}^{-2} u \mu^{\varepsilon} S_{d}^{-1}
\end{aligned}
$$

with $\varepsilon=4-d$. Here, $\mu$ is an external momentum scale, $t_{0 c}$ is a shift which for the results considered here can be set to zero, and $S_{d}$ stands for the surface of a $d$-dimensional hypersphere: $S_{d}=2^{1-d} \pi^{-d / 2} / \Gamma(d / 2)$. The $Z$-factors are determined by the condition that all poles at $\varepsilon=0$ are removed from the renormalized vertex functions.

The RG equations are written bearing in mind the fact that the bare vertex functions $\Gamma_{0}^{N, M}$ are calculated with the help of the bare Hamiltonian (2) as a sum of oneparticle irreducible (1PI) diagrams ${ }^{75}$ :

$$
\Gamma_{0}^{N, M}(\{r\},\{R\})=<\Psi_{0}\left(r_{1}\right) \ldots \Psi_{0}\left(r_{N}\right) A_{0}\left(R_{1}\right) \ldots A_{0}\left(R_{M}\right)>_{1 \mathrm{PI}}
$$

which do not depend on the scale $\mu$ and their derivatives with respect to $\mu$ at fixed bare parameters are equal to zero. So one gets

$$
\left.\mu \frac{\partial}{\partial \mu} \Gamma_{0}^{N, M}\right|_{0}=\left.\mu \frac{\partial}{\partial \mu} Z_{\Psi}^{-N / 2} Z_{A}^{-M / 2} \Gamma_{R}^{N, M}\right|_{0}=0
$$

where the index 0 means a differentiation at fixed bare parameters. So, the RG equations for the renormalized vertex function $\Gamma_{R}^{N, M}$ will be:

$$
\left(\mu \frac{\partial}{\partial \mu}+\beta_{u} \frac{\partial}{\partial u}+\beta_{f} \frac{\partial}{\partial f}+\zeta_{\nu} t \frac{\partial}{\partial t}-\frac{N}{2} \zeta_{\Psi}-\frac{M}{2} \zeta_{A}\right) \Gamma_{R}^{N, M}(t, u, f, \mu)=0
$$

where $f=e^{2}, \zeta_{\nu}=\zeta_{\Psi}-\zeta_{t}$ and the RG functions read

$$
\begin{array}{r}
\beta_{u}(u, f)=\left.\mu \frac{\partial u}{\partial \mu}\right|_{0}, \quad \beta_{f}(u, f)=\left.\mu \frac{\partial f}{\partial \mu}\right|_{0}, \\
\zeta_{\Psi}=\left.\mu \frac{\partial \ln Z_{\Psi}}{\partial \mu}\right|_{0}, \quad \zeta_{A}=\left.\mu \frac{\partial \ln Z_{A}}{\partial \mu}\right|_{0}, \quad \zeta_{t}=\left.\mu \frac{\partial \ln Z_{t}}{\partial \mu}\right|_{0} .
\end{array}
$$

Using the method of characteristics the solution of the RG equation may be written formally as:

$$
\Gamma_{R}^{N, M}(t, u, f, \mu)=X(l)^{N / 2}\left(X^{\prime}(l)\right)^{M / 2} \Gamma_{R}^{N, M}(Y(l) t, u(l), f(l), \mu l)
$$


where the characteristics are the solutions of the ordinary differential equations:

$$
\begin{aligned}
& l \frac{d}{d l} \ln X(l)=\zeta_{\Psi}(u(l), f(l)), \quad l \frac{d}{d l} \ln X^{\prime}(l)=\zeta_{A}(u(l), f(l)), \\
& l \frac{d}{d l} \ln Y(l)=\zeta_{\nu}(u(l), f(l)), \\
& l \frac{d}{d l} u(l)=\beta_{u}(u(l), f(l)), \quad l \frac{d}{d l} f(l)=\beta_{f}(u(l), f(l))
\end{aligned}
$$

with

$$
X(1)=X^{\prime}(1)=Y(1)=1, \quad u(1)=u, \quad f(1)=f .
$$

For small values of $l$, the equation (8) is mapping the large length scales (the critical region) to the noncritical point $l=1$. In this limit the scale-dependent values of the couplings $u(l), f(l)$ will approach the stable fixed point, if it exists.

The fixed points $u^{*}, f^{*}$ of the differential equations (10) are given by the solutions of the system of equations:

$$
\begin{aligned}
& \beta_{f}\left(u^{*}, f^{*}\right)=0, \\
& \beta_{u}\left(u^{*}, f^{*}\right)=0 .
\end{aligned}
$$

The stable fixed point is defined as the fixed point where the stability matrix

$$
B_{i j}=\frac{\partial \beta_{u_{i}}}{\partial u_{j}}, \quad u_{i}=\{u, f\}
$$

possess positive eigenvalues (or if complex, the eigenvalues with positive real parts). The stable fixed point corresponds to the critical point of the system. As we have mentioned above, in the limit $l \rightarrow 0$ (corresponding to the limit of an infinite correlation length) the renormalized couplings reach the values they have in the stable fixed point.

Now we can write the results for the RG functions obtained in a two-loop approximation $^{30}$ following the above described procedure in frames of the dimensional regularization and the minimal subtractions schemes. From a Ward identity one has $Z_{\Psi}=Z_{e}$, and the remaining $Z$-factors are to be found from the corresponding vertex functions $\Gamma^{2,0}, \Gamma^{0,2}$, and $\Gamma^{4,0}$. Since the gauge field is massless, the renormalization has been performed at a finite wave vector. The results in the two-loop order read:

$$
\begin{aligned}
& Z_{\Psi}=1+\frac{1}{\varepsilon}\left\{3 e^{2}-u^{2}(n+2) / 144+e^{4}[(n+18) / 4 \varepsilon-(11 n+18) / 48]\right\}, \\
& Z_{\mathrm{A}}=1+\frac{1}{\varepsilon}\left\{-n e^{2} / 6-n e^{4} / 2\right\} \\
& Z_{t}=1+\frac{1}{\varepsilon}\left\{(n+2) u / 6+u^{2}[(n+2)(n+5) / 36 \varepsilon-(n+2) / 24]+\right. \\
& \left.u e^{2}[-(n+2)(1 / 2 \varepsilon-1 / 3)]+e^{4}[(3 n+6) / 2 \varepsilon+(5 n+1) / 4]\right\}, \\
& Z_{u}=1+\frac{1}{\varepsilon}\left\{(n+8) u / 6+18 e^{4} / u+u^{2}\left[(n+8)^{2} / 36 \varepsilon-(5 n+22) / 36\right]+\right. \\
& u e^{2}[-(n+8) / 2 \varepsilon+(n+5) / 3]+e^{4}[(3 n+24) / \varepsilon+(5 n+13) / 2]+ \\
& \left.e^{6} / u[3(n+18) / \varepsilon-7 n / 2-45]\right\} .
\end{aligned}
$$

Following the standard procedure one then shows that the expressions for $\beta$ functions in the two-loop approximation will be: 


$$
\begin{aligned}
& \beta_{f}=-\varepsilon f+\frac{n}{6} f^{2}+n f^{3}, \\
& \beta_{u}=-\varepsilon u+\frac{n+8}{6} u^{2}-\frac{3 n+14}{12} u^{3}-6 u f+18 f^{2} \\
& +\frac{2 n+10}{3} u^{2} f+\frac{71 n+174}{12} u f^{2}-(7 n+90) f^{3} .
\end{aligned}
$$

The previous analysis of the equations of type (18), (19) either on a one-loop ${ }^{15}$ or two-loop level ${ }^{30}$ has been based on the direct solutions of the equation for the fixed point. In the present study we want to point out that the series have a zero radius of convergence and they are known to be asymptotic at best. Therefore, some additional mathematical methods have to be applied in order to obtain a reliable information on their basis.

We shall start by recalling the results of an $\varepsilon^{2}$-expansion for $\beta$-functions ${ }^{15,30}$. For the second order in $\varepsilon$ one obtains three fixed points: Gaussian $\left(u^{* G}=f^{* G}=0\right)$, "Uncharged" $\left(u^{* U} \neq 0, f^{* U}=0\right)$ and "Charged" $\left(u^{* C} \neq 0, f^{* C} \neq 0\right)$, to be denoted as $\mathrm{G}$, $\mathrm{U}, \mathrm{C}$. The expressions for them read:

$$
\begin{aligned}
& \mathrm{G}: \quad u^{* G}=0, \quad f^{* G}=0, \\
& \mathrm{U}: u^{* U}=u_{1}^{U} \varepsilon+u_{2}^{U} \varepsilon^{2}, f^{* U}=0, \\
& \mathrm{C}: u^{* C}=u_{1}^{C} \varepsilon+u_{2}^{C} \varepsilon^{2}, f^{* C}=f_{1}^{C} \varepsilon+f_{2}^{C} \varepsilon^{2},
\end{aligned}
$$

where

$$
\begin{aligned}
& u_{1}^{U}=\frac{6}{n+8}, \quad u_{2}^{U}=\frac{18(3 n+14)}{(n+8)^{3}}, \\
& u_{1}^{C}=\frac{3(n+36)+\left(n^{2}-360 n-2160\right)^{1 / 2}}{3 n(n+8)}, \\
& u_{2}^{C}=\frac{a_{2}}{a_{1}}, \quad f_{1}^{C}=\frac{6}{n}, \quad f_{2}^{C}=-\left(\frac{6}{n}\right)^{3} n,
\end{aligned}
$$

with

$$
\begin{aligned}
& a_{1}=1+\frac{n+8}{3} u_{1}^{C}-\frac{36}{n}, \quad a_{2}=\frac{3 n+14}{12}\left(u_{1}^{C}\right)^{3}-6 n u_{1}^{C}\left(\frac{6}{n}\right)^{3}+ \\
& 36 n\left(\frac{6}{n}\right)^{4}-\frac{(n+5) 4}{n}\left(u_{1}^{C}\right)^{3}-\frac{3(71 n+174)}{n^{2}} u_{1}^{C}+\left(\frac{6}{n}\right)^{3}(7 n+90) .
\end{aligned}
$$

Almost all physical results concerning the phase transition described by the field theory (2) are to some extent based on the information given by (20) - (22). The main ones read:

(i) the fixed point $\mathrm{U}$ is unstable with respect to the presence of an $f$-symmetry at $d<4$ with a stability exponent

$$
\lambda_{f}\left(u=u^{* U}, f=f^{* U}=0\right)=\left.\frac{\partial \beta_{f}}{\partial f}\right|_{U}=-\varepsilon
$$




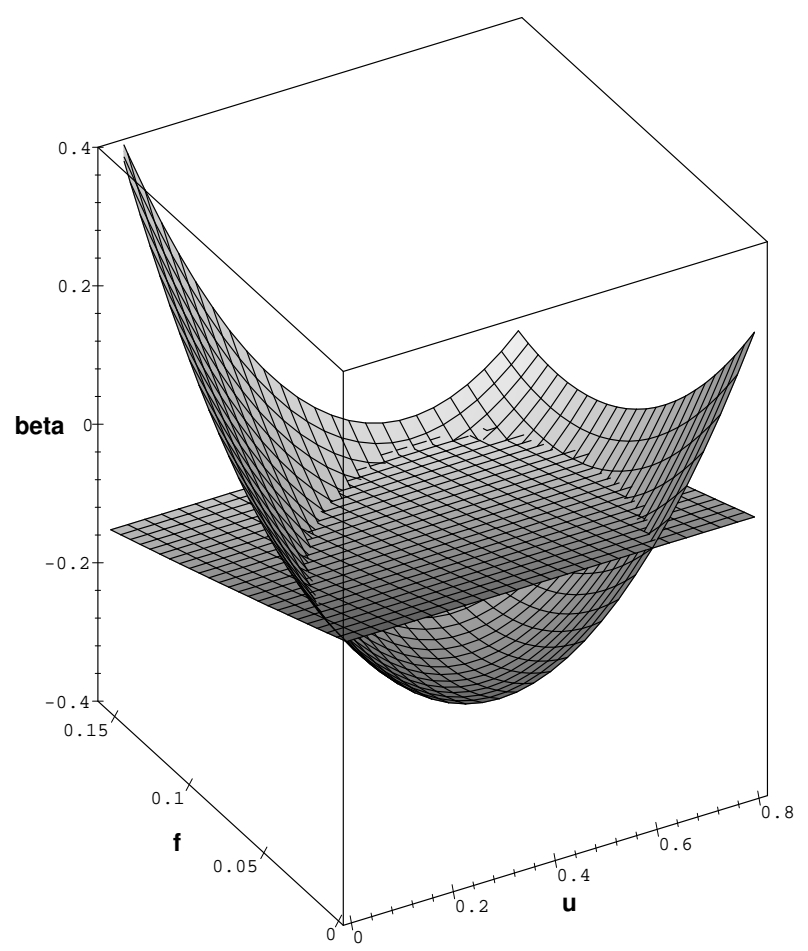

Figure 1. $\beta$-functions of the model of superconductor $\beta_{u}(u, f), \beta_{f}(u, f)$ in one-loop approximation for $d=3, n=2$. The fixed points have coordinates $\left(u^{*}=f^{*}=0\right),\left(u^{*}=0.6, f^{*}=0\right)$.

(ii) the fixed point $\mathrm{C}$ appears to be complex for $n<n_{c}=365.9$ already $^{15}$ on a one-loop level. The stability exponent is given by

$$
\lambda_{u}\left(u=u^{* C}, f=f^{* C}\right)=\left.\frac{\partial \beta_{u}}{\partial u}\right|_{C}
$$

and on the two-loop level it reads:

$$
\lambda_{u}=-\varepsilon s, \quad s=\left[\left(1+\frac{36}{n}\right)^{2}-\frac{432(n+8)}{n^{2}}\right]^{1 / 2}
$$

leading to an oscillatory flow of $u$ in a one-loop order below $n_{c}$ with the soluti$\mathrm{on}^{26,30}$ :

$$
\begin{aligned}
& f(l)=\frac{6 f l^{-\varepsilon}}{6+n \varepsilon f\left(l^{-\varepsilon}-1\right)}, \\
& u(l)=f(l) \frac{n}{2(n+8)}\left\{s \operatorname { t a n } \left[\frac{s}{2} \ln \left(f(l) f^{-1} l^{\varepsilon}\right)\right.\right. \\
& \left.\left.+\arctan \left(\frac{2(n+8)}{s n} \frac{u}{f}+\frac{n+36}{n s}\right)\right]-\frac{n+36}{n}\right\}
\end{aligned}
$$

here $f$ and $u$ are the initial parameters for $l=1$;

(iii) from the condition of the positiveness of the fixed point coordinate $f^{*}\left(f=e^{2}\right)$ it follows that at $\varepsilon=1, n$ has to be larger that 36. This questions the applicability of the $\varepsilon$-expansion for $n=2$ to $d=3$. 


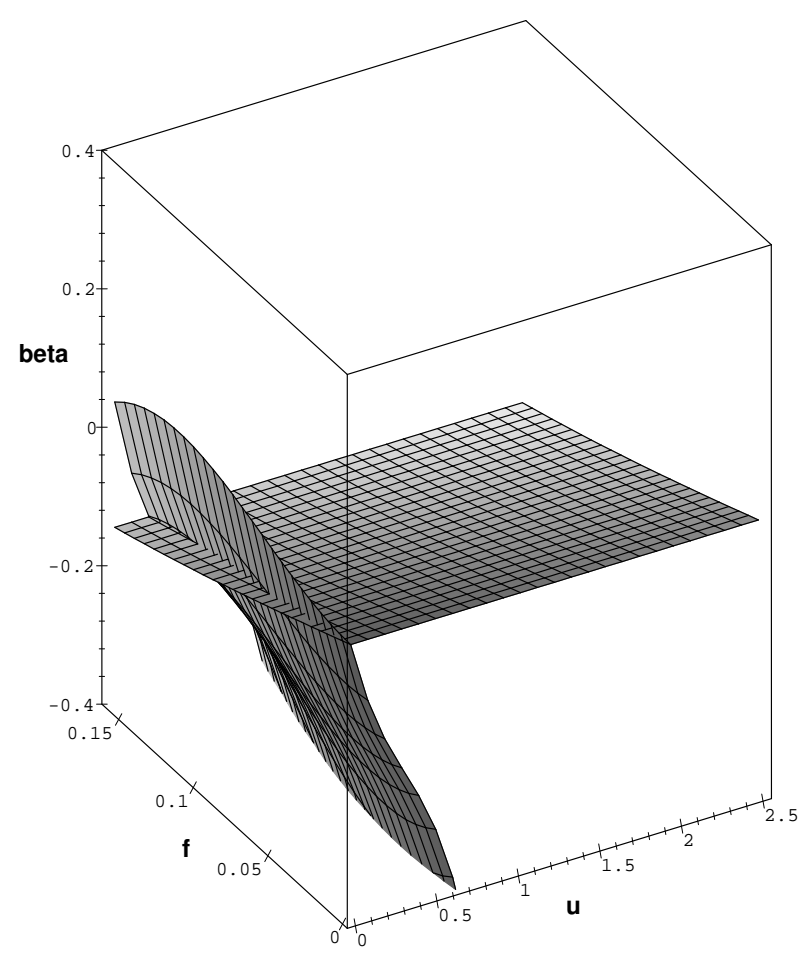

Figure 2. $\beta$-functions of the model of superconductor $\beta_{u}(u, f), \beta_{f}(u, f)$ in two-loop approximation for $d=3, n=2$. Only the Gaussian fixed point $u^{*}=f^{*}=0$ survives.

So, it follows that for the "superconductor" case $n=2$ which is the most interesting from a physical point of view a stable fixed point does not exist and, therefore, the observed phase transition is of a first order.

Now we will study the RG equations in the minimal subtraction scheme in the framework of $d=3$ theory $^{11,12,13}$ putting $\varepsilon=1$ in expressions for the RG functions and studying the perturbation theory in powers of coupling constants. The latter corresponds to the number of loops in Feynman diagrams and one develops, therefore, the perturbation theory in a successive number of loops. Direct calculations based on equations (18), (19) at fixed $d=3$ do not bring qualitatively new features to the described above analysis. In the one-loop approximation, leaving square terms in (18), (19) one finds that only one nontrivial fixed point $u^{*}=6 /(n+8), f^{*}=0$ exists. The $\beta$-functions $\beta_{u}(u, f), \beta_{f}(u, f)$ in the one-loop approximation at $d=3, n=2$ are shown in the Fig. 1. The simultaneous intersection of the surfaces corresponding to both functions with the plane $\beta=0$ results in the fixed points; for the $n=2$ they have coordinates $u^{*}=f^{*}=0$ and $u^{*}=0.6, f^{*}=0$ as seen in the picture. In the two-loop approximation only the Gaussian fixed point survives, as one may see from Fig. 2 .

Nevertheless one should note that such a straightforward interpretation of the above expansions data has been questioned and the way of analyzing the series for $\beta$-functions (18), (19) avoiding the strict $\varepsilon$-expansion and exploiting the information on the accurate solution for the pure model at $d=3$, has been proposed ${ }^{30}$. Also, from the comparison of $\varepsilon$-expansion data for $f^{*}$ (giving positive values of $f^{*}$ only for $n>36$ ) with the value of $f^{*}$ obtained without the $\varepsilon$-expansion (remaining positive for all $n$ ) it has been conjectured that the lower boundary for $n$ resulting in the negative $f^{*}$ may be an artifact of the expansion procedure. Let us now consider expressions for the RG functions more carefully, paying attention to their possible asymptotic nature and 
treating them with a resummation procedure.

\section{RESUMMATION}

The resummation technique appropriate for critical phenomena and applied to the asymptotic series for the RG functions enables one to obtain extremely accurate values of the critical exponents ${ }^{76}$. In fact, the asymptotic nature of the series for the RG functions has only been proven in the case of the $\phi^{4}$ model containing one coupling of an $O(n)$-symmetry (the $n$-vector model). The high-order asymptotics for these series are known $^{27,28,29}$ in analytical form as well. These results give the possibility of obtaining precise values for the critical exponents of the $n$-vector model by a resummation of the corresponding series for the renormalization group functions (see e.g. ${ }^{7,8,11}$ ). As far as we know, no information similar to that obtained in ${ }^{27,28,29}$ for the "uncharged" case $(f=0)$ is available for the "charged" model we are considering here. For models containing several couplings of different symmetries the asymptotic nature of the corresponding series for the RG functions is a generally accepted belief rather than a proven fact.

As it will be important in the course of our future analysis we shall mention here the weakly diluted $n$-vector model, describing the ferromagnetic ordering in a system of $N_{1}$ classical $n$-component "spins" located in $N$ sites of a lattice $\left(N_{1} / N<1\right)$ and quenched in a certain configuration. Using the replica trick in order to perform the quenched averaging one concludes ${ }^{82}$ that an effective Hamiltonian of such a model contains two fourth order terms of a different symmetry and reads:

$$
H=\int \mathrm{d}^{d} x\left\{\frac{1}{2} \sum_{\alpha=1}^{m}\left[\left|\nabla \overrightarrow{\phi^{\alpha}}\right|^{2}+m_{0}^{2}\left|\vec{\phi}^{\alpha}\right|^{2}\right]-\frac{v_{0}}{8}\left(\sum_{\alpha=1}^{m}\left|\vec{\phi}^{\alpha}\right|^{2}\right)^{2}+\frac{u_{0}}{4 !} \sum_{\alpha=1}^{m}\left(\left|\vec{\phi}^{\alpha}\right|^{2}\right)^{2}\right\}
$$

where $\vec{\phi}^{\alpha}$ is an $n$-component vector $\vec{\phi}^{\alpha}=\left(\phi^{\alpha, 1}, \phi^{\alpha, 2}, \ldots, \phi^{\alpha, n}\right) ; u_{0}>0, v_{0}>0$ are bare coupling constants; $m_{0}$ is the bare mass and in the final results a replica limit $m \rightarrow 0$ is to be taken. The RG functions for this model are obtained in the form of a double series in renormalized couplings $u, v$ and the asymptotic nature of the series has not been proven for this model up till now ${ }^{84}$. Nevertheless, the appropriate resummation technique (applied as if these series are asymptotic ones) enables one to obtain accurate values for critical exponents in three dimensions ${ }^{77,78,79,80,81}$ and to describe (in the $n=1$ case) the experimentally observed crossover to a new type of a critical behavior caused by the weak dilution ${ }^{85,86}$. These results have been confirmed by Monte-Carlo ${ }^{87,} 88$ and Monte-Carlo $\mathrm{RG}^{89}$ calculations.

The two main ways of a resummation commonly used for the asymptotic series arising in the RG approach are: (i) a resummation based on the conformal mapping technique and (ii) the Padé-Borel resummation. Case (i) is based on the conformal transformation, which maps part of the analytical domain containing the real positive axis onto a circle centered at the origin and the asymptotic expansion for a certain function is thus re-written in the form of a new series $\left(\mathrm{see}^{8}\right)$. This resummation, however, is based on the knowledge of subtle details of asymptotics (the location of the pole, the high-order behavior) which are not available in our case.

In the absence of knowledge about the singularities of the series, the most appropriate method which can be used to perform the analytical continuation is the Padé approximation resulting in the Padé-Borel resummation technique (ii) (see e.g. ${ }^{7}$ ). We are going to apply it to the special case of $f=0$, so let us disucss it in detail. 
Starting from the Taylor series for the function $f(u)$ :

$$
f(u)=\sum_{j \geq 0} c_{j} u^{j}
$$

one constructs the Borel-Leroy transform

$$
F(u t)=\sum_{j \geq 0} \frac{c_{j}}{\Gamma(j+p+1)}(u t)^{j}
$$

with $\Gamma(x)$ the Euler's gamma-function and $p$ some positive number ${ }^{90}$. Then one represents (27) in the form of the Padé approximant $F_{[L / M]}^{\text {Padé }}(u t)$ :

$$
F_{[L / M]}^{\text {Padé }}(x)=\frac{\sum_{i=0}^{L} a_{i} x^{i}}{\sum_{j=0}^{M} b_{j} x^{j}}
$$

(in the subsequent analysis, proceeding in a two-loop approximation we will use the [1/1] Padé approximant) and the resummed function will be given by:

$$
f^{\operatorname{Res}}(u)=\int_{0}^{\infty} d t e^{-t} t^{p} F^{\text {Padé }}(u t) .
$$

The resummation scheme (27) - (29) of the (asymptotic) series in one variable (26) is easily generalized to the two-variable case when the series is given in a form:

$$
f(u, v)=\sum_{j, j \geq 0} c_{i, j} u^{i} v^{j}
$$

with the Borel-Leroy transform

$$
F(u, v, t)=\sum_{i, j \geq 0} \frac{c_{i, j}}{\Gamma(i+j+p+1)}(u t)^{i}(v t)^{j} .
$$

The procedure is aimed to help how to choose an appropriate form of the analytic continuation of the series (31). Two most common methods to proceed are the Borel resummation combined with Chisholm approximants and the Borel resummation of the resolvent series, presented in a form of the Padé approximant. For the first method, in order to write an analytic continuation of the series (31) one uses the rational approximants of two variables: so-called Canterbury approximants or a generalized Chisholm approximants $^{91,93}$ which are a generalization of Padé approximants in the case of several variables, representing (31) in a form:

$$
F^{\text {Chisholm }}(u, v, t)=\frac{\sum_{i, j} a_{i, j} u^{i} v^{j} t^{i+j}}{\sum_{i, j} b_{i, j} u^{i} v^{j} t^{i+j}}
$$

(sums in the numerator and denominator are limited by the condition of the correspondence between known numbers of terms in the initial series and that in the approximant). Again, the resummed function is given by an integral (29):

$$
f^{\operatorname{Res}}(u, v)=\int_{0}^{\infty} d t e^{-t} t^{p} F^{\text {Chisholm }}(u t) .
$$




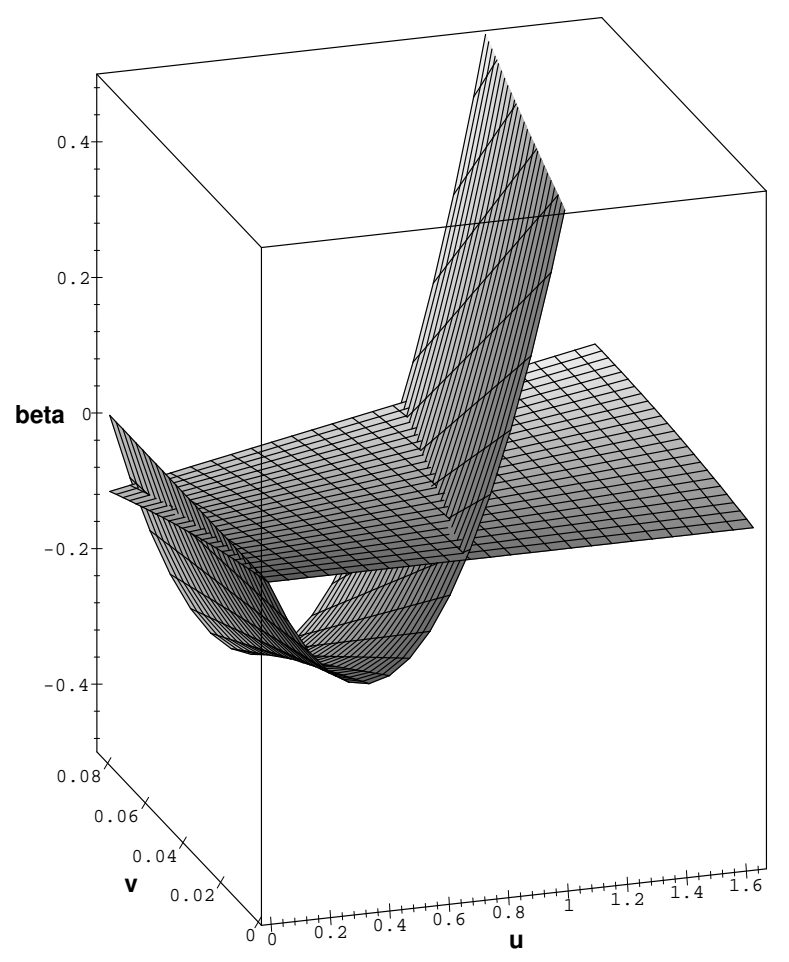

Figure 3. $\beta$-functions of the diluted Ising model $\beta_{u}(u, v), \beta_{v}(u, v)$ in one-loop approximation for $d=3$. The fixed points have coordinates $\left(u^{*}=v^{*}=0\right),\left(u^{*}=0.667, v^{*}=0\right)$.

Proceeding with the second method, one writes for the series of two variables (30) the so-called resolvent series $\mathcal{F}(u, v, \tau)^{92,93}$ introducing an auxiliary variable $\tau$, which allows the separation of contributions from different orders of the perturbation theory in the variables $u, v$ :

$$
\begin{aligned}
\mathcal{F}(u, v, \tau) & =\sum_{i, j \geq 0} c_{i, j}(u \tau)^{i}(v \tau)^{j}, \\
f(u, v) & =\mathcal{F}(u, v, \tau=1) .
\end{aligned}
$$

Now the resummation of the series $\mathcal{F}(u, v, \tau)$ is performed with respect to the variable $\tau$ as for the series in a single variable, applying the above described scheme (27) - (29).

Let us illustrate how the resummation procedure works in the case of the effective Hamiltonian (25). In order to make a direct comparison with the superconductor case, let us take the $\beta$-functions obtained for the model (25) in the minimal subtraction scheme in the two-loop approximation, though high-order results are available for this model $^{81,95}$ as well as results $77,78,79,80$ obtained in the $d=3$ massive field theoretical approach $^{94}$. The expressions for the $\beta$-functions, corresponding to the renormalized couplings $u, v$ in the replica limit $m \rightarrow 0$ for the Ising model $(\mathrm{n}=1)$ read:

$$
\begin{aligned}
& \beta_{u}=-\varepsilon u+\frac{3}{4} u^{2}-6 u v-\frac{17}{12} u^{3}+\frac{23}{2} u^{2} v-\frac{41}{2} u v^{2}, \\
& \beta_{v}=-\varepsilon v+u v+-4 v^{2}-\frac{5}{12} u^{2} v+\frac{11}{2} u v^{2}-\frac{21}{2} v^{3} .
\end{aligned}
$$

We shall not present the expressions for the other RG functions here, as we are going to study only the fixed point equations. 
Looking for the solutions of the fixed point equations for functions (35), (36) one can show that in the one-loop approximation, in addition to the Gaussian fixed point $u^{*}=v^{*}=0$, there exist two more solutions $u^{*}=2 / 3, v^{*}=0$ and $u^{*}=0, v^{*}=-1 / 5$ and the solution $u^{*} \neq 0, v^{*} \neq 0$ is absent ${ }^{96}$. The fixed point with $v^{*}<0$ is beyond the region of parameters describing the diluted magnet ${ }^{97}$ and the pure model fixed point $u^{*} \neq 0, v^{*}=0$ appears to be unstable with respect to the $v$-coupling (we propose to the reader to check this by looking at the stability matrix $B_{i j}(u, v)$ (13) eigenvalues at the fixed points). The corresponding plot of the functions $\beta_{u}, \beta_{v}$ in the one-loop approximation is shown in the figure 3. Passing to the two-loop approximation makes the result even worse: only the Gaussian fixed point is present (see Fig. 田). Returning back to the initial problem statement one should conclude that the obtained picture corresponds to the absence of a second order phase transition in a $d=3$ Ising model with a weak dilution as well as without a dilution (the absence of the fixed point $\left.u^{*} \neq 0, v^{*}=0\right)$. Of course, this is contrary to the real situation. Let us further note that the behaviour obtained for the $\beta$-functions of the model (25) in the one- and twoloop approximations (Figs. 3, 44) resembles those for the superconductor case in the corresponding approximations (Figs. 1, 2).

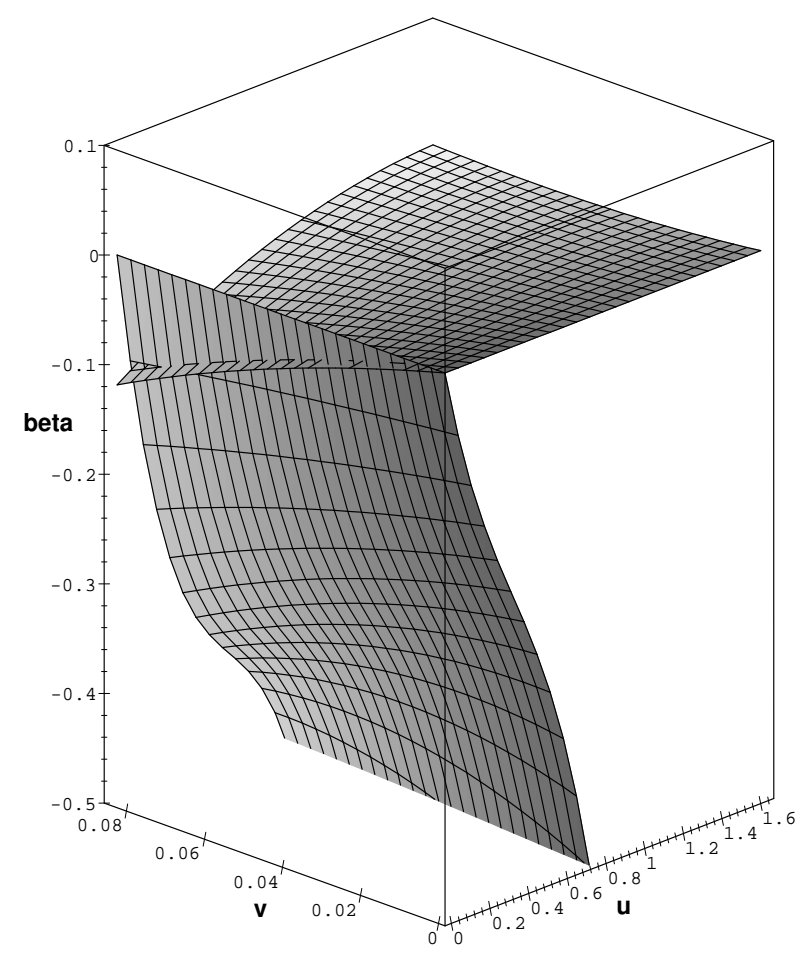

Figure 4. $\beta$-functions of the diluted Ising model $\beta_{u}(u, v), \beta_{v}(u, v)$ in two-loop approximation for $d=3$. Only the Gaussian fixed point $u^{*}=v^{*}=0$ survives.

Applying, however, the resummation procedure to the series (35), (36) in the two loop approximation one reconstitutes fixed points $\left(u^{*} \neq 0, v^{*}=0\right),\left(u^{*}=0, v^{*} \neq 0\right)$ and obtains a new stable fixed point $u^{*} \neq 0, v^{*} \neq 0$ which governs a second order phase transition in a weakly diluted Ising model. The picture obtained appears to be stable with respect to successive accounts of the higher order terms in the perturbation theory, when the appropriate resummation technique is applied. As we have already claimed above, these RG results are confirmed by other different theoretical approaches and correspond to the experimentally observed second order phase transition in the 
weakly diluted Ising magnet with critical exponents differing from those of the pure case. In Fig. 5 we show the crossing of the $\beta_{u}(u, v)$ and $\beta_{v}(u, v)$ surfaces for the resummed function. The calculations have been performed by means of the Padé-Borel resummation technique for the resolvent series (34) of two-loop functions (35), (36) as described above ${ }^{98}$. The Gaussian $\left(u^{*}=v^{*}=0\right)$ and pure $\left(u^{*}=1.3146, v^{*}=0\right)$ fixed points can be seen at the rear of the cube. The cross-section of $u$ and $v$ planes in the picture are chosen to pass through the stable fixed point $\left(u^{*}=1.6330, v^{*}=0.0835\right)$ corresponding to a new critical behaviour.

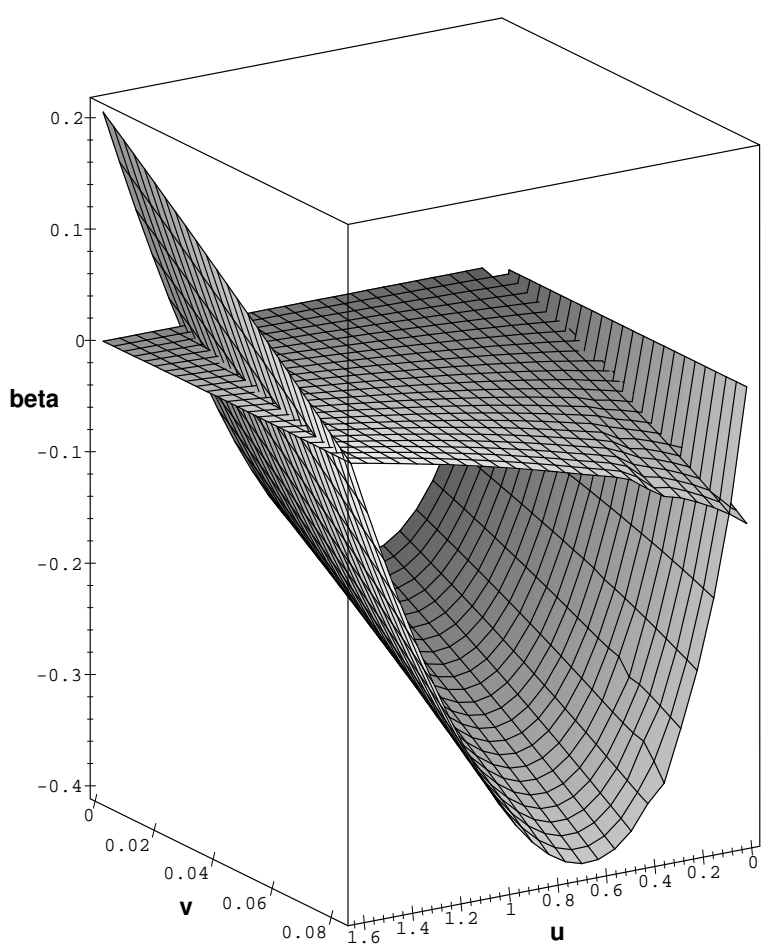

Figure 5. $\beta$-functions of the diluted Ising model $\beta_{u}(u, v), \beta_{v}(u, v)$ in two-loop approximation for $d=3$ obtained by applying Padé-Borel resummation technique. Resummation restores the presence of the fixed point $u^{*} \neq 0, v^{*}=0$ and results in the appearance of a new stable fixed point $u^{*} \neq 0, v^{*} \neq 0$.

The example we have considered above is a typical situation happening in a $d=$ 3 RG theory: when considered without an appropriate resummation technique, the RG analysis might not only give imprecise values for critical exponents but also a qualitatively wrong answer about the absence of a stable fixed point for a certain model, resulting in the absence of a second order phase transition. Now with this information in hand let us pass to the analysis of a model of a superconductor described in the two-loop approximation by the RG functions (35), (36).

\section{FIXED POINTS AND FLOWS IN THREE DIMENSIONS}

We shall continue here by considering the flow equations (10) directly for $d=3$. We shall look for the solutions of the fixed point equations at $d=3$ paying attention to the possible asymptotic nature of the corresponding series (18), (19). Consider first the equation for the uncharged fixed point $U$. Substituting $f^{*}=0$ into (19) one obtains 
the following expression for the function $\beta_{u}^{U} \equiv \beta_{u}\left(u, f^{*}=0\right)$ :

$$
\beta_{u}^{U}=-u+\frac{n+8}{6} u^{2}-\frac{3 n+14}{12} u^{3}
$$

Solving this polynomial for the fixed point one obtains for the non-trivial $u^{*}>0$ :

$$
u^{* U}=\frac{n+8}{3 n+14}+\frac{\sqrt{n^{2}-20 n-104}}{3 n+14}
$$

and immediately the "condition of the existence of a non-trivial solution $u^{* U " ~ q u a l i t a-~}$ tively very similar to this, appearing in the frame of the $\varepsilon$-expansion technique, follows ( see $^{15,30}$ and formula (21) of the present article as well): the solution exists only for certain values of $n>n_{c}=24.3$ !

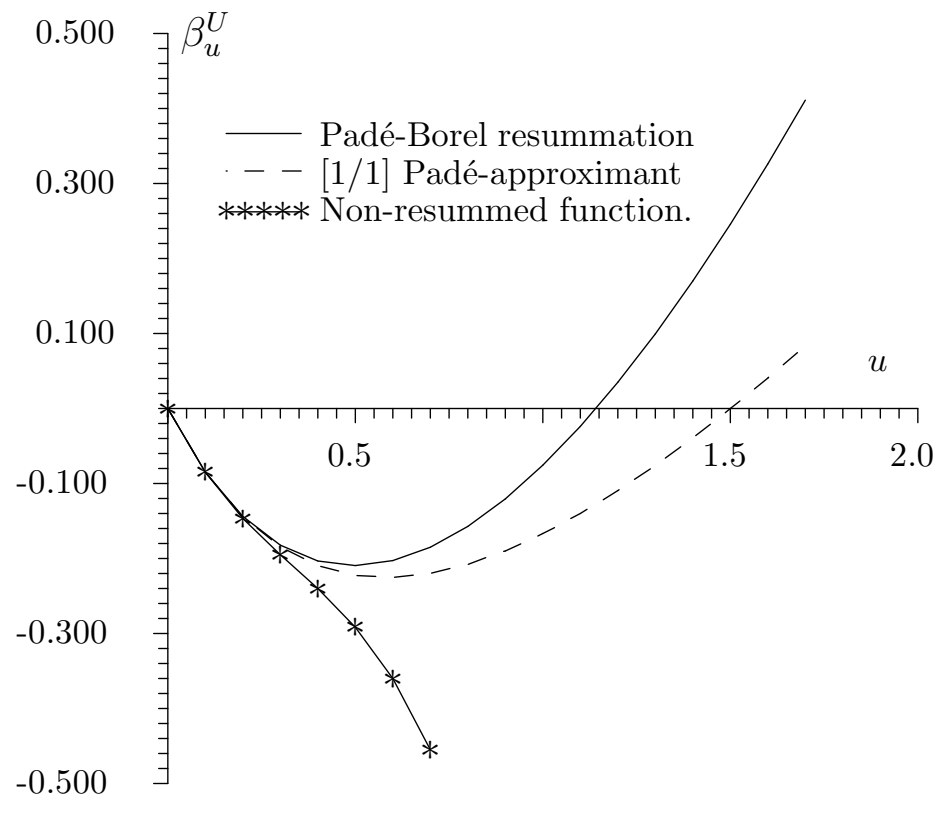

Figure 6. $\beta_{u}$-function of the uncharged model $\beta_{u}^{U}$ at $d=3, n=2$.

From Fig. 6 one can see that the function $\beta_{u}^{U}$ (37) does not intersect the $u$-axis for any non-zero value of $u$ for $n=2$. In the $O(n)$-symmetric $\phi^{4}$-theory at $d=3$ this situation is well known (see e.g. ${ }^{94,99}$ ). The $\beta$-function calculated directly at $d=3$ does not possess a stable zero for the realistic values of $n$, nevertheless with in the three-loop order the presence of the stable fixed point is restored. To avoid this artifact appearing in the two-loop calculation one can either resum the series for the $\beta$-function or construct the appropriate Padé approximant ${ }^{100}$ in order to perform the analytical continuation of (37) out of the domain of convergence (which is equal to zero for the series in the right-hand side of (37)). Let us try both methods. Representing (37) in the form of a [1/1] Padé approximant:

$$
\beta_{u}^{U, \text { Padé }}=u \frac{-1+A_{u} u}{1+B_{u} u}
$$

one obtains:

$$
A_{u}=\frac{n^{2}+7 n+22}{6(n+8)}, \quad B_{u}=\frac{3 n+14}{2(n+8)}
$$


Table 1. The fixed point $\mathrm{U}$ coordinate $u^{* U}$ as a function of $n . u^{* U, \text { Padé: }}$ obtained on the basis of the [1/1] Padé approximant; $u^{* U, R e s}$ : obtained by the Padé-Borel resummation.

\begin{tabular}{lllllllll}
\hline \hline$n$ & 1 & 2 & 3 & 4 & 5 & 6 & 7 & 8 \\
\hline$u^{* U, \text { Padé }}$ & 1.800 & 1.500 & 1.269 & 1.091 & 0.951 & 0.840 & 0.750 & 0.676 \\
$u^{* U, \text { Res }}$ & 1.315 & 1.142 & 1.002 & 0.888 & 0.794 & 0.717 & 0.652 & 0.597 \\
\hline \hline
\end{tabular}

and, solving the equation for the fixed point:

$$
\beta_{u}^{U, \text { Padé }}\left(u^{* P, \text { Padé}}\right)=0
$$

one obtains:

$$
u^{* U, \text { Padé }}=\frac{6(n+8)}{n^{2}+7 n+22} .
$$

So we have obtained a qualitatively different situation. The behavior of the function $\beta_{u}^{U, \text { Padé }}(u)$ for $n=2$ is shown in Fig. 6 by the dashed curve. If one is interested in more accurate values of $u^{*}$ some resummation has to be applied. Choosing the Padé-Borel resummation technique ${ }^{101}$ and following the scheme $(26)-(29)$ one obtains for the resummed function $\beta_{u}^{U, \operatorname{Res} 98}$ :

$$
\beta_{u}^{U, R e s}=u\left[2\left(1-A_{u} / B_{u}\right)\left(1-E\left(\frac{2}{u B_{u}}\right)\right)-1\right]
$$

the coefficients $A_{u}, B_{u}$ are given by (40), $E(x)=x e^{x} E_{1}(x)$, where the function

$$
E_{1}(x)=e^{-x} \int_{0}^{\infty} d t e^{-t}(x+t)^{-1}
$$

is connected with the exponential integral by the relation ${ }^{102}$ :

$$
E_{1}(x \pm i 0)=-E i(-x) \mp i \pi
$$

The behavior of the function $\beta_{u}^{U, \text { Res }}(u)$ is shown in Fig. 6 by the solid curve and the fixed point coordinate $u^{* U, R e s}$ is obtained solving the non-linear equation:

$$
\beta_{u}^{U, \operatorname{Res}}\left(u^{* U, R e s}\right)=0 .
$$

The coordinates of the fixed point $u^{* U}$ obtained on the basis of the Padé approximation

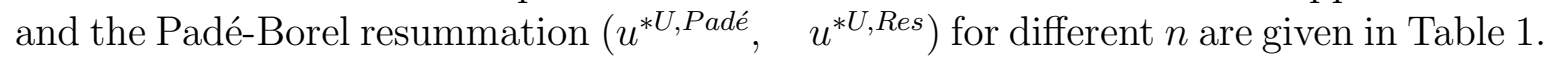

From this analysis we conclude that: in the $d=3$ theory the Padé approximants (as an analytical continuation of $\beta$-functions) may change the picture qualitatively and lead to values of fixed points comparable to those obtained by the Padé-Borel resummation technique.

Consider now the equation for the charged fixed point $C$ applying the above considerations to $\beta_{f}$, for which the expression at $d=3$ reads (18):

$$
\beta_{f}=-f+\frac{n}{6} f^{2}+n f^{3}
$$




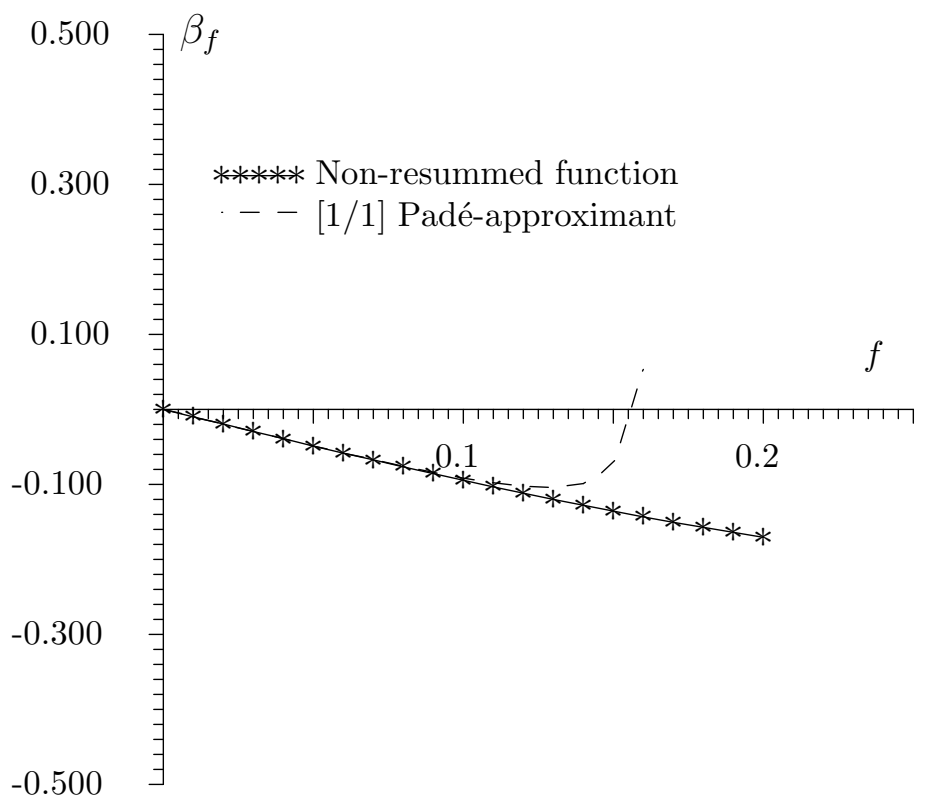

Figure 7. $\beta_{f}$-function at $d=3, n=2$.

The behavior of $\beta_{f}$ as a function of $f$ is shown in Fig. 7 by asterisks. Note, however, that in this case the function $\beta_{f}$ even without any resummation possesses a non-trivial zero $f^{* M}$ (its value $f^{* C, D i r}$ is given in the 2 nd row of Table 2). Representing (45) in the form of the [1/1] Padé approximant:

$$
\beta_{f}^{\text {Padé }}=f \frac{-1+A_{f} f}{1+B_{f} f}
$$

one has for $A_{f}, B_{f}$ :

$$
A_{f}=\frac{n+36}{6}, \quad B_{f}=-6,
$$

and, solving the equation for the fixed point coordinate $f^{* C, \text { Padé: }}$

$$
\beta_{f}^{\text {Padé }}\left(f^{* C, \text { Padé}}\right)=0
$$

one obtains:

$$
f^{* C, \text { Padé }}=\frac{6}{n+36} .
$$

The function $\beta_{f}^{\text {Padé }}(f)$ is shown in Fig. 7 by the dashed line, the coordinate $f * C$, Padé is given in the 3rd row of Table 2. But now the series (45) is not alternating and this results in the presence of a pole (at $f=1 / 6$ ) in the approximant (46). Therefore, (46) correctly represents the function $\beta_{f}(f)$ only for $f<1 / 6$. Let us note however that for all positive $n$ a fixed point exists and its coordinate $f^{* M, \text { Padé }}$ lies within the limits $0<f^{* C \text {,Padé }}<1 / 6$, where there are no poles in (46). Comparing this result with that obtained for the uncharged fixed point one can note that the representation of $\beta_{f}$ in the form of the Padé approximant does not qualitatively change the picture (a solution for $\beta_{f}(f)=0$ exists at $d=3$ even without an analytical continuation) but results in a decrease of the fixed point coordinate. Contrary to the $\varepsilon$-expansion values (22) there are no borderline values of $n$ for the positivity of $f^{* C}$. Unfortunately, we cannot check this result by means of the Padé-Borel resummation technique. The above 
Table 2. The fixed point $\mathrm{C}$ coordinate $f^{* C}$ as a function of $n . f^{* C, D i r}$ : obtained by a direct solution of the equation for the fixed point; $f^{* C \text {,Padé: }}$ obtained on the basis of the $[1 / 1]$ - Padé approximant; $f^{* C, \varepsilon}$ : the $\varepsilon$-expansion result with the linear accuracy in $\varepsilon ; f^{* C, \varepsilon^{2}}$ : the $\varepsilon$-expansion result with the square accuracy in $\varepsilon$.

\begin{tabular}{lllllllll}
\hline \hline$n$ & 1 & 2 & 3 & 4 & 5 & 6 & 7 & 8 \\
\hline$f^{* C, \text { Dir }}$ & 0.920 & 0.629 & 0.500 & 0.424 & 0.372 & 0.333 & 0.304 & 0.280 \\
$f^{* C, \text { Padé }}$ & 0.162 & 0.158 & 0.154 & 0.150 & 0.146 & 0.143 & 0.140 & 0.136 \\
$f^{* C, \varepsilon}$ & 6.000 & 3.000 & 2.000 & 1.500 & 1.200 & 1.000 & 0.857 & 0.750 \\
$f^{* C, \varepsilon^{2}}$ & -210.000 & -51.000 & -22.000 & -12.000 & -7.440 & -5.000 & -3.551 & -2.625 \\
\hline \hline
\end{tabular}

mentioned presence of a pole in the denominator of the Padé approximant makes the corresponding integral representation problematic ${ }^{103}$. In order to find the $u$-coordinate of the fixed point $\mathrm{C}, u^{* C}$, we have to deal with a function of two variables, $\beta_{u}(u, f)$, represented by a rather short series (19). Another problem arises due to the fact that the function $\beta_{u}(u, f)$ contains generating terms (i.e. $\beta_{u}(u=0, f) \neq 0$ ). In order to perform some kind of an analytic continuation of the function of two variables one can use the Chisholm approximants (32 $)^{91,93}$. But, the presence of generating terms makes this choice rather ambiguous. The most reliable way in such case is the representation of $\beta_{u}(u, f)$ in the form of a resolvent series $B(u, f, \tau)(34)^{92,93}$ introducing an auxiliary variable $\tau$, which allows the separation of contributions from different orders of the perturbation theory in the coupling constants. The series for $B(u, f, \tau)$ then reads:

$$
B(u, f, \tau) \equiv \beta_{u}(u \tau, f \tau)=\sum_{j \geq 0} b_{j} \tau^{j}
$$

with the usual notation applied for the coefficients $b_{j}$. Now one considers (50) as a series in the single variable $\tau$. This series can be represented in the form of the Padé approximant $B^{\text {Padé }}(u, f, \tau)$ as an analytical continuation of the function $B(u, f, \tau)$ for a general value of $\tau$. In particular at $\tau=1$ the equality holds $B(u, f, \tau=1)=\beta_{u}(u, f)$ and the approximant

$$
B^{\text {Padé }}(u, f, \tau=1) \equiv \beta_{u}^{\text {Padé }}(u, f)
$$

represents the initial function $\beta_{u}(u, f)$. In our case the expression for $B(u, f, \tau)$ reads:

$$
B(u, f, \tau)=\tau\left(b_{1}+b_{2} \tau+b_{3} \tau^{2}\right)
$$

where:

$$
\begin{gathered}
b_{1}=-u, \quad b_{2}=\frac{n+8}{6} u^{2}-6 u f+18 f^{2}, \\
b_{3}=-\frac{3 n+14}{12} u^{3}+\frac{2 n+10}{3} u^{2} f+\frac{71 n+174}{12} u f^{2}-(7 n+90) f^{3} .
\end{gathered}
$$




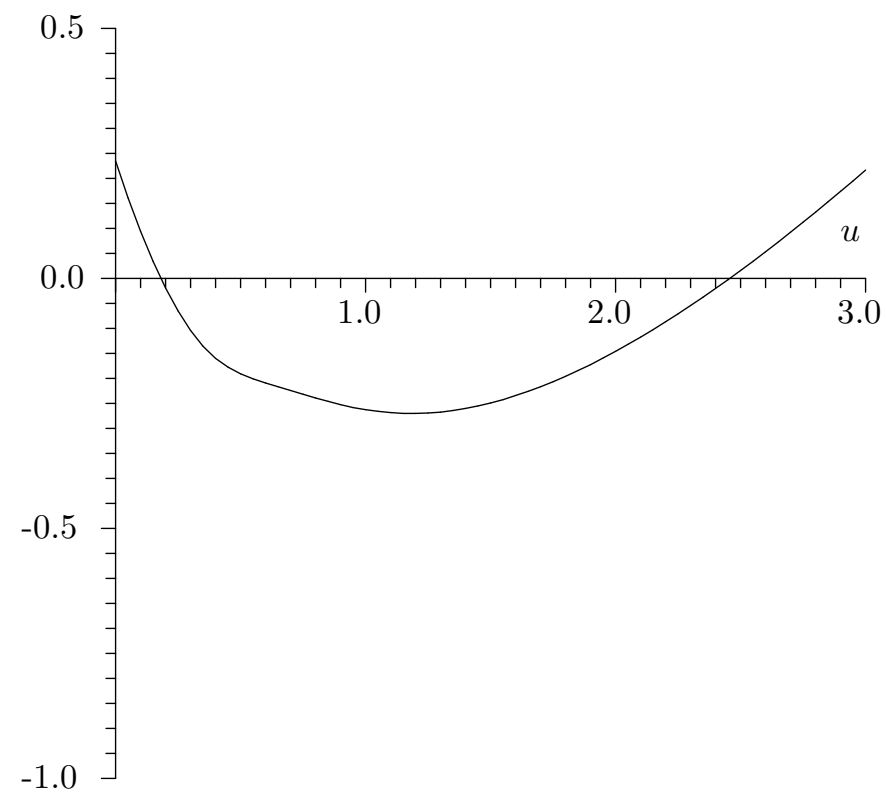

Figure 8. Intersection of the function $\beta_{u}^{\text {Padé }}(u, f)$ at $d=3, n=2$ with the plain $f=f^{* C, \text { Padé }}$ in two-loop approximation.

Table 3. The fixed point $\mathrm{C}$ coordinates $u^{* C, P a d e ́}$ obtained on the basis of the [1/1] Padé approximant for the "resolvent" series as a function of $n$. C1 : the unstable fixed point; $\mathrm{C} 2$ : the stable fixed point.

\begin{tabular}{lllllllll}
\hline \hline$n$ & 1 & 2 & 3 & 4 & 5 & 6 & 7 & 8 \\
\hline $\mathrm{C} 1$ & 0.184 & 0.181 & 0.179 & 0.177 & 0.175 & 0.175 & 0.176 & 0.179 \\
$\mathrm{C} 2$ & 3.309 & 2.457 & 1.781 & 1.150 & 0.473 & 0.369 & 0.305 & 0.256 \\
\hline \hline
\end{tabular}

Representing the expression in brackets in the right-hand side of (51) in the form of a [1/1] Padé approximant we have:

$$
B^{\text {Padé }}(u, f, \tau)=\tau b_{1} \frac{1+A_{u, f} \tau}{1+B_{u, f} \tau}
$$

where

$$
A_{u, f}=\frac{b_{2}}{b_{1}}-\frac{b_{3}}{b_{2}}, \quad B_{u, f}=\frac{-b_{3}}{b_{2}} .
$$

Let us note here that the function $B(u, f, t)$ obtained in this way as the approximant for the function of two variables $\beta_{u}(u, f)$ obeys certain projection properties in the single-variable case: substituting $f=0$ or $u=0$ into (53) one obtains the [1/1] Padé approximant for $\beta_{u}^{U}(u)$ or the [0/1] Padé approximant for $\beta_{u}(u=0, f)$. Finally the expression for $\beta_{u}(u, f)$ approximated in such way reads:

$$
\beta_{u}^{\text {Padé }}(u, f)=b_{1} \frac{1+A_{u, f}}{1+B_{u, f}} .
$$

Substituting into the equation for the fixed point $\beta_{u}\left(u^{* C}, f^{* C}\right)=0$ the value for the coordinate $f^{* C}=f^{* C \text {,Padé }}(49)$ one obtains the non-linear equation for $u^{* C, \text { Padé: }}$ 


$$
\beta_{u}^{\text {Padé }}\left(u, f=f^{* C, \text { Padé }}\right)=0 .
$$

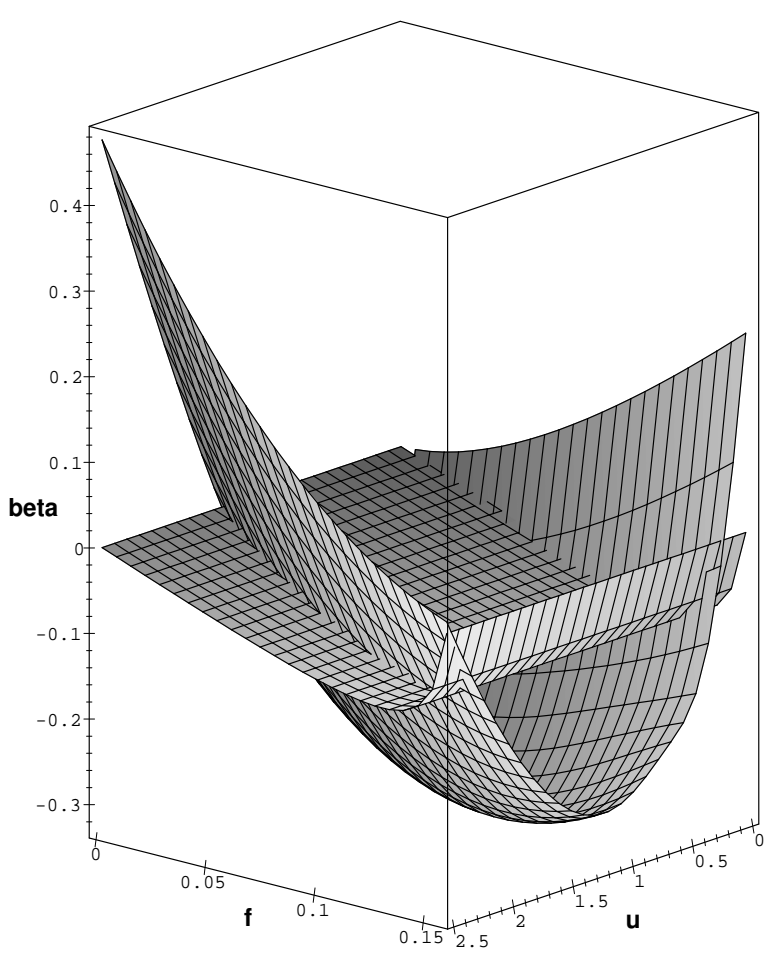

Figure 9. $\beta$-functions of the model of superconductor $\beta_{u}(u, f), \beta_{f}(u, f)$ in two-loop approximation for $d=3, n=2$ obtained by Padé analysis for the resolvent series. The stable "charged" fixed point $\mathrm{C} 2$ with coordinates $u^{*}=2.457, f^{*}=0.158$ as well as the unstable fixed point C1

$u^{*}=0.181, f^{*}=0.158$ are seen on the front side of the cube. Gaussian $\left(u^{*}=f^{*}=0\right)$ and "uncharged" $\left(u^{*}=1.500, f^{*}=0\right)$ fixed points are located on the rear of the cube.

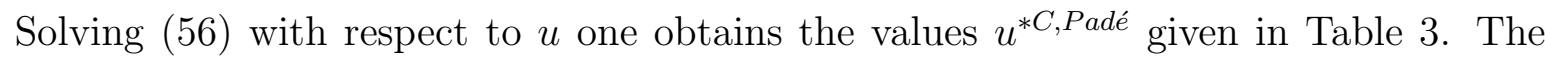

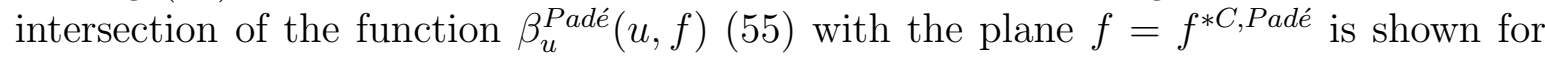
$n=2$ in Fig. 8. The first fixed point (C1) given in the 2 nd row of Table 3 turns out to be unstable, while the fixed point $\mathrm{C} 2$ is stable in the case $n=2$ we are predominantly interested in.

The resulting picture of $\beta$-functions surfaces is shown in the Fig. 9 . The Gaussian and uncharged fixed points may be seen at the rear of the picture, whereas the intersection of the $u$ - and $f$-planes has been chosen in the picture to cross the stable fixed point $\mathrm{C} 2$. The unstable fixed point $\mathrm{C} 1$ is seen as well.

The crossover to the asymptotic critical behavior is described by the solutions of the flow equations (10) with the initial values of $u\left(\ell_{0}\right)$ and $f\left(\ell_{0}\right)$ at $\ell=\ell_{0}{ }^{104}$. Substituting the analytical continuation of the $\beta$-functions in the right-hand side of (10), with the Padé approximants (46), (55) we get the following system of differential equations:

$$
\begin{aligned}
& l \frac{d f}{d l}=f \frac{-1+A_{f} f}{1+B_{f} f} \\
& l \frac{d u}{d l}=-u \frac{1+A_{u, f}}{1+B_{u, f}}
\end{aligned}
$$

where $A_{f}, B_{f}$ and $A_{u, f}, B_{u, f}$ are given by (47) and (54), respectively. 


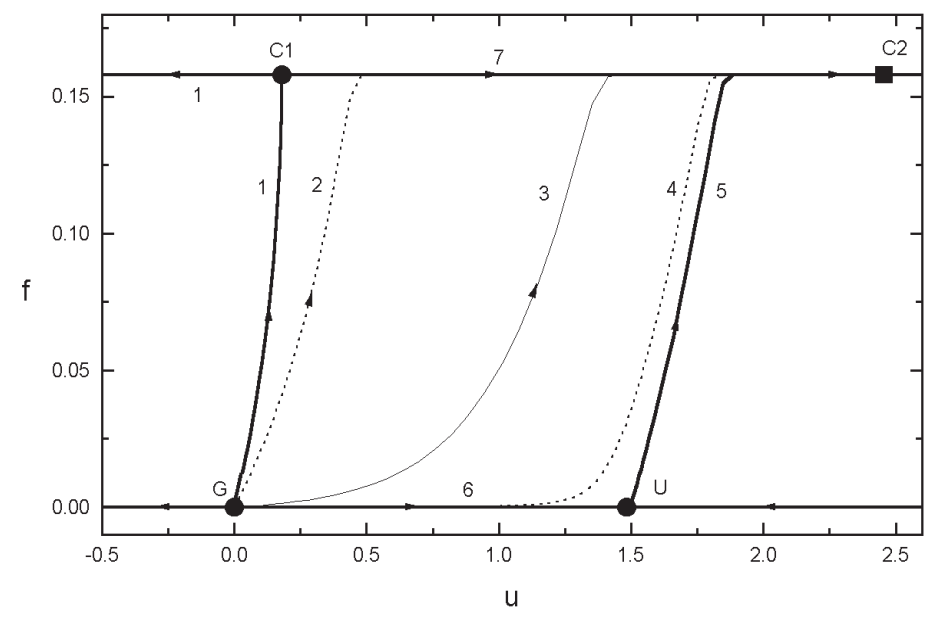

Figure 10. Flow lines for the case $n=2, d=3$ given by equations (57), (58). Fixed points $\mathrm{G}, \mathrm{U}$, $\mathrm{C} 1$ are unstable, fixed point $\mathrm{C} 2$ (shown by a box) is a stable one (for further description see text).

Solving equations (57), (58) numerically one obtains the flow diagram shown in Fig. 10 for the case $n=2$. The space of the couplings is divided into several parts by separatrices (thick lines in Fig. 10) connecting the fixed points. Besides the Gaussian $(\mathrm{G})$ there exist three fixed points, one corresponding to the uncharged (U) and two others corresponding to the charged $(\mathrm{C} 1, \mathrm{C} 2)$ cases. The fixed points $\mathrm{G}, \mathrm{C} 1$ and $\mathrm{U}$ are unstable (solid circles in Fig. 10) and the fixed point C2 is stable (shown as a solid box in Fig. 10). Several different flow lines are shown in Fig. 10. They can be compared with the corresponding flow picture obtained by a direct solution of the flow equations for the two-loop $\beta$-functions expressed by the third-order polynomials in couplings $u$, $f(18),(19)$ (see Fig. $2 \mathrm{a} \mathrm{in}^{30}$ ). There, one can see that no stable fixed point exist and furthermore that the fixed point $\mathrm{U}$ is absent. Comparing Fig. 10 and Fig. $2 \mathrm{~b}$ from $^{30}$ one can see how an analytical continuation of the $\beta$-functions (10), (18), done only partly in ${ }^{30}$ and performed here in the form of Padé approximants restores the presence of the fixed point $\mathrm{U}$ (unstable) and leads to the appearance of a new stable fixed point $\mathrm{C} 2$ for the charged model. The coordinates of the fixed points $\mathrm{U}, \mathrm{C} 1, \mathrm{C} 2$ are given in the corresponding rows of Tables [1, 2, 通 and for $n=2$ they are equal to:

$$
\begin{aligned}
& \mathrm{U}: u^{*}=1.500, f^{*}=0, \\
& \mathrm{C} 1: u^{*}=0.181, f^{*}=0.158, \\
& \mathrm{C} 2: u^{*}=2.457, f^{*}=0.158 .
\end{aligned}
$$

\section{CRITICAL EXPONENTS}

Values of the critical exponents can be determined by the fixed point values of the $\zeta$-functions defined on the basis of renormalizing $Z$-factors (14) - (17) by:

$$
\zeta_{i}=\mu \partial \ln Z_{i} / \partial \mu,
$$


where the derivative is taken at fixed unrenormalized couplings. The expressions for the $\zeta$-functions related to the order parameter and the temperature field renormalization in the two-loop approximation read $^{30}$ :

$$
\begin{aligned}
\zeta_{\psi}= & -3 f+\frac{(n+2)}{72} u^{2}+\frac{(11 n+18)}{24} f^{2}, \\
\zeta_{t}= & \frac{-(n+2)}{6} u+\frac{(n+2)}{12} u^{2}- \\
& \frac{2(n+2)}{3} u f-\frac{(5 n+1)}{2} f^{2}, \\
\zeta_{A}= & \frac{n}{6} f+n f^{2} .
\end{aligned}
$$

If there exists a stable fixed point, the critical exponent of the correlation length $\nu$, of the order parameter susceptibility $\gamma$ and of the specific heat $\alpha$ will be given by:

$$
\begin{aligned}
\nu & =\left(2-\zeta_{\nu}^{*}\right)^{-1}, \\
\gamma & =\left(2-\zeta_{\nu}^{*}\right)^{-1}\left(2-\zeta_{\psi}^{*}\right), \\
\alpha & =\left(2-\zeta_{\nu}^{*}\right)^{-1}\left(\varepsilon-2 \zeta_{\nu}^{*}\right), \\
\eta & =\zeta_{\psi}^{*},
\end{aligned}
$$

where $\zeta_{\nu}=\zeta_{\psi}-\zeta_{t}$. The exponents $(63)$ - 66 are related by the familiar scaling laws. From the analysis above it follows that the charged fixed point $C 2$ is stable and this results in values for exponents (63)-(65) that are different from the values of the uncharged fixed point U, i.e. they are not given by the ${ }^{4} \mathrm{He}$ values as it is sometimes stated (see e.g. ${ }^{16,59,25}$ ).

Recently, an interesting consequence of the existence of a stable charged fixed point $\left(C_{2}\right)$ has been observed ${ }^{62}$. According to the charge renormalization (3) the $\beta_{f}$-function reads

$$
\beta_{f}=f\left(\varepsilon-\zeta_{A}(f, u)\right) .
$$

Thus at a fixed point with a nonzero $f^{*}$ the value of the gauge field $\zeta$-function is given by $\zeta_{A}^{*}=\varepsilon$ exactly. This means that the penetration depth $\lambda$ and the correlation length $\xi$ are proportional and the temperature dependence follows a power law with the exponent $\nu^{62}$. This is not the case, though, at the fixed point with $f^{*}=0$. There we have $\zeta_{A}^{*}=0$ (each loop contribution to the $\zeta_{A}$-function contains at least one $f$-factor) and the penetration depth behaves as $\lambda \sim \xi^{(2-\varepsilon) / 2}$. Thus one would have two different critical length scales.

Now, trying to obtain numerical values of the critical exponents on the basis of the

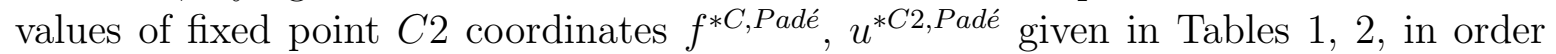
to be self-consistent let us perform the same type of an analytical continuation for the $\zeta$-functions series as those which have applied to the $\beta$-functions (18), (19). So, after introducing the auxiliary variable $\tau$ let us represent functions (63) - (65) in the form of resolvent series in $\tau$ and then chose the [1/1] Padé approximants for these series, which at $\tau=1$ will give us the analytical continuation of the series requested. Thus, the expression for the critical exponent $\phi(\phi \equiv\{\nu, \gamma, \alpha)\}$ reads:

$$
\phi=a_{\phi}^{(0)} \frac{1+A_{\phi}}{1+B_{\phi}} .
$$

The expressions for the coefficients $A_{\phi}, B_{\phi}$ in (68) read:

$$
A_{\phi}=a_{\phi}^{(1)}+B_{\phi} ; \quad B_{\phi}=-a_{\phi}^{(2)} / a_{\phi}^{(1)}
$$


and $a_{\phi}^{(i)}$ are to be determined from the resolvent series in $\tau$ :

$$
\phi=\left.\sum_{i \geq 0} a_{\phi}^{(i)} \tau^{i}\right|_{\tau=1}
$$

Substituting (60) and (61) into (63) - (65) and representing (63) - (65) in the form of (70) one finds:

$$
\begin{aligned}
& a_{\nu}^{(0)}=1 / 2, \\
& a_{\nu}^{(1)}=(n+2) / 12 u-3 / 2 f, \\
& a_{\nu}^{(2)}=\left(n^{2}-n-6\right) / 144 u^{2}+(71 n+138) / 48 f^{2}+(n+2) / 12 u f, \\
& a_{\gamma}^{(0)}=1, \\
& a_{\gamma}^{(1)}=(n+2) / 12 u, \\
& a_{\gamma}^{(2)}=\left(n^{2}-2 n-8\right) / 144 u^{2}+(5 n+1) / 4 f^{2}+5(n+2) / 24 u f, \\
& a_{\alpha}^{(0)}=1 \\
& a_{\alpha}^{(1)}=-3(n+2) / 12 u+9 / 2 f, \\
& a_{\alpha}^{(2)}=\left(-3 n^{2}+3 n+18\right) / 144 u^{2}-(71 n+138) / 16 f^{2}- \\
& (n+2) / 4 u f .
\end{aligned}
$$

Now considering the case $n=2$ and substituting coordinates of the fixed point $C 2$ $\left(f^{* C, \text { Padé }}=.158\right), u^{* C 2, \text { Padé }}=2.457$ (see Tables 1, 2) into $(71)-(73)$ one obtains for the critical exponents $(63)-(66)^{105}$ :

$$
\begin{aligned}
& \nu=0.86, \quad \gamma=1.88 \\
& \alpha=-1.14, \quad \eta=-0.19
\end{aligned}
$$

The application of the Padé approximants for the analytical continuation of the functions may result in the appearance of poles in these functions. If the pole is located in a region of the expansion parameters which has no physical meaning, e.g. a negative coupling $u$ or $f$, the analysis is not complicated. This is the case for the $\beta$-functions in the region of couplings less than the fixed point values. For the $\zeta$-functions, however, considering the non-asymptotic behavior (and thus being far from the stable fixed point) one passes through a region of couplings where the Padé approximation for the $\zeta$-functions becomes ambiguous which results in the appearance of a pole. Therefore, studying the crossover behavior in the next subsection we will still keep the polynomial representation for $\zeta$-functions instead of the Padé approximants. Then for the asymptotic values of critical exponents one gets:

$$
\begin{aligned}
& \nu=0.77, \quad \gamma=1.62 \\
& \alpha=-0.31, \quad \eta=-0.10
\end{aligned}
$$

The comparison of the values (75) and (76) shows a numerical difference of $15 \%$ in $\nu$ and $\gamma$ and a considerable increase of $\alpha$ but there is no qualitative change (e.g. the sign of the specific heat exponent remains the same). This should be compared with values given by others, $\nu=0.53$ and $\eta=-0.70^{62}$ and $\eta=-0.38^{61}$. Since for the conventional superconductors the experimentally accessible regime lies in the precritical region further away from $T_{c}$, let us now discuss some non-asymptotic quantities such as effective exponents and amplitude ratios. 


\section{AMPLITUDE RATIO FOR THE SPECIFIC HEAT}

One of the most interesting measurable quantities is the specific heat. Near a second order phase transition, asymptotically it follows a power law

$$
C_{0}^{ \pm}=\frac{A^{ \pm}}{\alpha}|t|^{-\alpha}+\text { const }
$$

where \pm indicates the specific heat $C$ and its non universal amplitude $A$ above and below $T_{c}$. The amplitude ratio $A^{+} / A^{-}$found from the ratio $C^{+}\left(t^{+}\right)_{0} / C^{-}\left(t^{-}\right)_{0}$ after subtracting the non singular background value constitutes a universal quantity at $T_{c}$ depending only on the dimension and the number of components of the order parameter.

The calculation of this ratio can be extended to the non asymptotic region ${ }^{106,107}$ resulting in a temperature dependent measurable quantity. This also tests the description of the nonasymptotic behavior by a certain flow in the interaction space of the Hamiltonians, discussed for the effective exponents. The starting point in the calculation is the renormalization group equation for the specific heat $C^{ \pm}$

$$
\left[\mu \frac{\partial}{\partial \mu}+\beta_{u} \frac{\partial}{\partial u}+\beta_{f} \frac{\partial}{\partial f}+\zeta_{\nu}\left(2+t \frac{\partial}{\partial t}\right)\right] C^{ \pm}(t, u, f, \mu)=\mu^{-\varepsilon} B(u, f),
$$

where the inhomogeneity $B$ comes from the additive renormalization. The formal solution reads

$$
\begin{aligned}
& C^{ \pm}(t, u, f, \mu)=\mu^{-\varepsilon} \exp \left[-\int_{1}^{l}\left(\varepsilon-2 \zeta_{n} u(x) \frac{d x}{x}\right]\right. \\
& \times\left\{F^{ \pm}(l)-\int_{1}^{l} \frac{d y}{y} B(y) \exp \left[-\int_{l}^{y}\left(\varepsilon-2 \zeta_{\nu}(x)\right) \frac{d x}{x}\right]\right\} .
\end{aligned}
$$

The amplitude ratio is most easily calculated by choosing the same value for the flow parameter both above and below $T_{c}$, which means $t^{+}=-2 t^{-108}$. We then recover the asymptotic expression found in ${ }^{107}$

$$
\frac{A^{+}}{A^{-}}=2^{\alpha} \frac{B \nu+F^{+} \alpha}{B \nu+F^{-} \alpha}
$$

where the functions $B$ and $F^{ \pm}$are taken at the fixed point. For this function we use the lowest order result known from the $\Psi^{4}$ theory neglecting the coupling to the gauge field; $B=2 n, F^{+}=-n$ and $F^{-}=12 / u^{*}-4$. Then we have for $n=2$

$$
\frac{A^{+}}{A^{-}}=2^{\alpha} \frac{2 \nu-\alpha}{2 \nu-2 \alpha+6 \alpha / u^{*}} .
$$

In the Table 1 we have collected the values obtained for the different fixed points. It is interesting to note the reasonable estimate for this ratio presented in $^{109}$ for the superfluid phase transition. The authors have found a value of $A^{+} / A^{-}=1.067$, which is surprisingly near the value obtained in the stable charged fixed point using both 
Table 4. Asymptotic values for the specific heat amplitude ratio at various fixed points. The exponents in the first two lines correspond to the procedure leading to (75). The third and the fourth lines correspond to the procedure leading to (76).

\begin{tabular}{ccccc}
\hline \hline F.P. & $A^{+} / A^{-}$ & $\alpha$ & $\nu$ & $u^{*}$ \\
\hline $\mathrm{U}$ & 1.81 & -0.33 & 0.72 & 1.500 \\
$\mathrm{C} 2$ & 1.07 & -1.14 & 0.86 & 2.457 \\
$\mathrm{U}$ & 0.78 & 0.15 & 0.62 & 1.500 \\
$\mathrm{C} 2$ & 1.06 & -0.31 & 0.77 & 2.457 \\
\hline \hline
\end{tabular}

calculation schemes, although the exponents are very different. We have not, however, taken into account changes in the scaling functions due to the coupling $f$.

In comparison with experimental results ${ }^{18}$, the amplitude ratio of the Gaussian $n$-vector model without a coupling to the gauge field $A^{+} / A^{-}=n / 2^{3 / 2}$ has been used since the dimension of the order parameter is unclear. Later on this expression for the amplitude ratio has been calculated for cases other than the isotropic symmetry. This leads to a dependence of the ratio on the higher order couplings ${ }^{110}$.

\section{EFFECTIVE EXPONENTS}

Effective exponents are usually defined by the logarithmic temperature derivatives of the corresponding correlation functions (see e.g. ${ }^{26,111}$ ). They can be found from the solutions of the renormalization group equation for the renormalized vertex functions. The effective exponents contain two contributions, one from the corresponding $\zeta$-functions now taken at values of $u(\ell), f(\ell)$ of the particular flow curve considered ("the exponent part"), and one from the change of the corresponding scaling function ("the amplitude part"). For the analysis below, we neglect the latter contributions since we expect them to be smaller than the differences for the fixed point values of the exponents coming from the different treatments discussed before. Thus we have:

$$
\begin{aligned}
\nu & =\left(2-\zeta_{\nu}(\ell)\right)^{-1}, \\
\gamma & =\left(2-\zeta_{\nu}(\ell)\right)^{-1}\left(2-\zeta_{\psi}(\ell)\right), \\
\alpha & =\left(2-\zeta_{\nu}(\ell)\right)^{-1}\left(\varepsilon-2 \zeta_{\nu}(\ell)\right) .
\end{aligned}
$$

The flow parameter $\ell$ can be related to the relative temperature distance $T_{c}$ by the matching condition $t(\ell)=\left(\xi_{0}^{-1} \ell\right)^{2}$, with $\xi_{0}$ the amplitude of the correlation length.

We have computed these effective exponents, see Fig. 11 - Fig. 13, along the flow lines shown in Fig. 10 by inserting ${ }^{112}$ values of the couplings $u(\ell)$ and $f(\ell)$ into Eqs. (81) - (83). For the separatrix 1 we have started with initial conditions leading to a flow, which does not coincide with the fixed point $\mathrm{C} 1$ but slightly misses it although the flow curve does not differ from the separatrix within the thickness of the lines shown in Fig. 


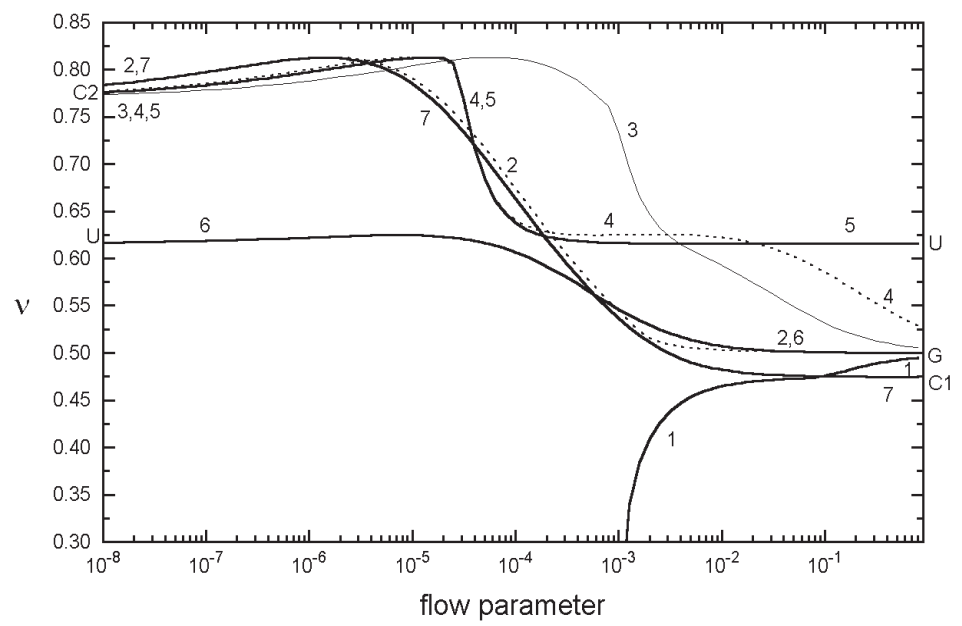

Figure 11. Effective exponent $\nu$ for the flows shown in Fig.10 (for further description see text).

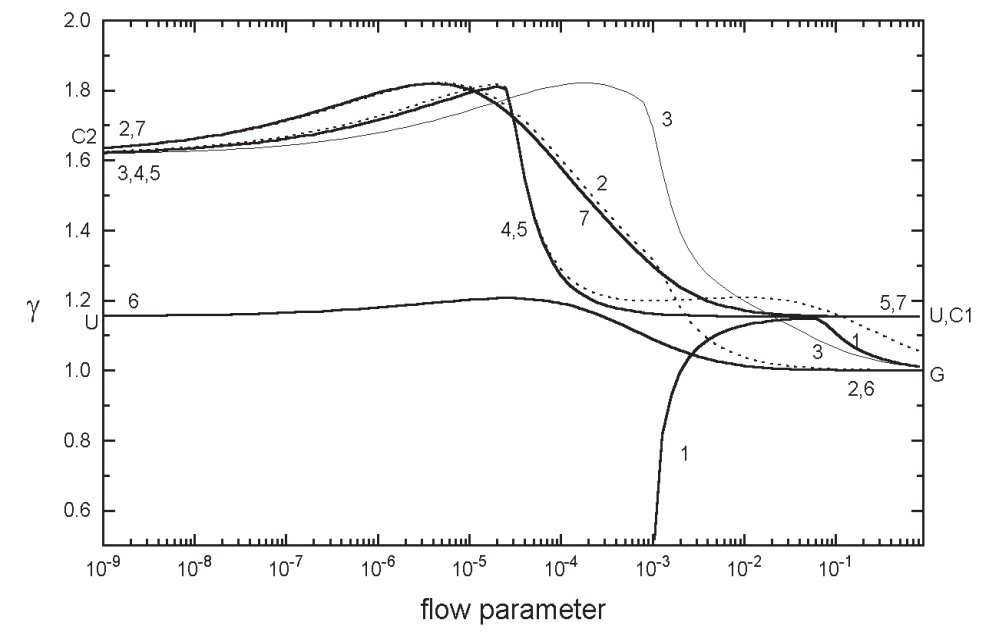

Figure 12. Effective exponent $\gamma$ for the flows shown in Fig.10 (for further description see text). 


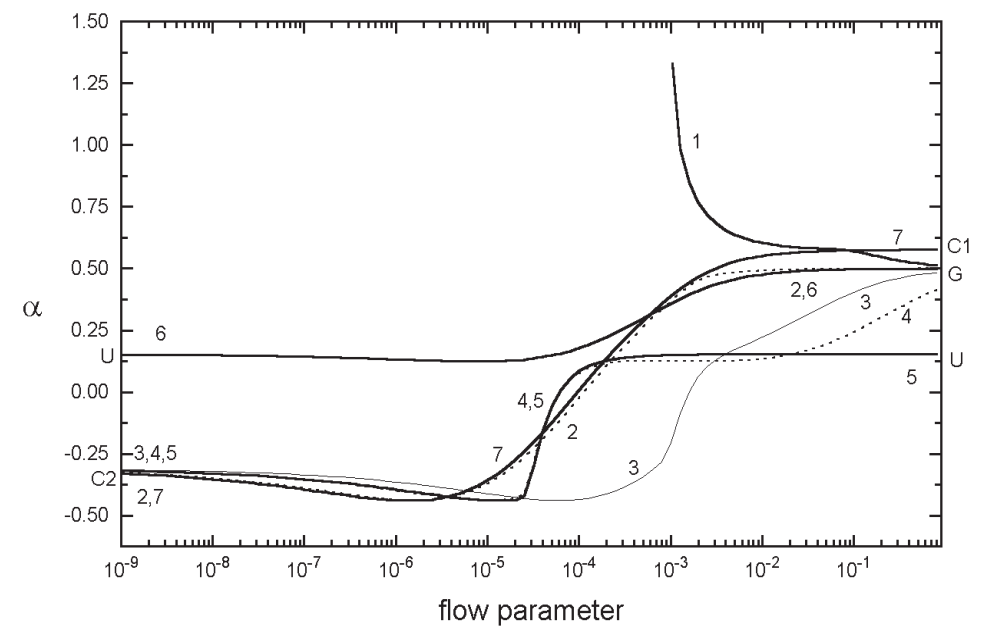

Figure 13. Effective exponent $\alpha$ for the flows shown in Fig.10 (for further description see text).

10. For the curve number 4, we start somewhat further away from the Gaussian fixed point $\mathrm{G}$ leading to the initial values of the effective exponents between their Gaussian values and their values for the uncharged fixed point U. Note that when the values of the effective exponent $\gamma$ for the uncharged fixed point $U$ and the charged fixed point $\mathrm{C} 1$ are the same within the accuracy given by the scale of the figure.

Note that when the coupling $f$ to the gauge field fluctuations is small (i.e. for extreme type-II superconductors) the RG flow passes very closely to the uncharged fixed point $U$ and the effective exponents, and within some region of temperatures, they coincide with those of the uncharged superfluid liquid. In this region the effective Hamiltonian (2) may be considered as that of a superconductor in a constant magnetic field neglecting magnetic field fluctuations. Recently for such a model it has been shown that near the zero-field critical point the singular part of the free energy scales as $F_{\text {sing }} \simeq|t|^{2-\alpha} \mathcal{F}\left(B|t|^{-2 \nu}\right)$ with $\nu$ being the coherence length exponent ${ }^{113}$.

\section{CONCLUSIONS}

Does the above account give a definite conclusion about the order of the phase transition occurring in a model of the superconductor minimally coupled to the gauge field? First of all one should keep in mind that such an answer may be given in an analytical theory framework only by obtaining an exact result or a rigorous proof. Here, the problem has been treated by a perturbation theory approach and the account of the influence of fluctuations on the order of the phase transition is studied within the field theoretical RG technique. We have shown that remaining within this approach one may get an answer about the second order phase transition occurring in the above mentioned model.

The main point discussed in this context is whether the equations for $\beta$-functions possess a stable fixed point or not. The absence of the stable fixed point is often interpreted as a change of the order of the phase transition (caused by the presence of the magnetic field fluctuations) and the evidence of the fluctuation-induced first-order 
phase transition. This change of the order of the phase transition (being of the secondorder in the absence of the coupling to the gauge), however, is confirmed only by the perturbation theory calculations in low orders ${ }^{15}$, $\left(\mathrm{see}^{30}\right.$ and the references therein as well).

Applying a simple Padé analysis to the series under discussion ${ }^{114}$ we have shown how one can recover a stable fixed point in the RG equations. In the case of one coupling, such an approach gives a qualitatively correct picture of the phase transition and restores the presence of a stable fixed point $\left({ }^{94}\right.$, see formulas (38), (42) of this article as well). The same occurs in the case of two couplings: for $n=2$ the "uncharged" fixed point $\mathrm{U}$ (having coordinates $f^{* U, \text { Padé }}=.158, u^{* U, \text { Padé }}=2.457$ ) appears to be stable, which leads to a new set of critical exponents. We should note, however, that the pair correlation function critical exponent $\eta$, calculated by familiar scaling relations on the basis of sets of values (75) or (76), remains negative which agrees with the result of ${ }^{61,62,54}$. Being calculated only in a two-loop approximation with the application of the Padé analysis, these values for the critical exponents are to be considered as preliminary ones. The main point we wish to make is that within the framework of the renormalization group analysis for the superconductor model there remains the possibility of a second-order phase transition characterized by a set of critical exponents differing from those of ${ }^{4} \mathrm{He}$. Another important task could be to calculate the nonasymptotic specific heat in order to compare with experiments within the region of the crossover to the background.

\section{ACKNOWLEDGEMENTS}

We acknowledge the useful discussions with A.M.J. Schakel, D.I. Uzunov, A.E. Filippov. We are grateful to O.J. Poole who read the manuscript and made suggestions for improving the text.

\section{REFERENCES}

1. K. G. Wilson, Phys. Rev. B 4:3174 (1971); ibid 4:3184 (1971).

2. $\quad$ N. N. Bogoliubov, D. V. Shirkov. "Introduction to the Theory of Quantized Fields," Wiley \& Sons, New York (1959).

3. D. J. Amit. "Field Theory, the Renormalization Group, and Critical Phenomena," World Scientific, Singapore (1984).

4. M. Le Bellac. "Quantum and Statistical Field Theory," Claredon Press, Oxford (1991).

5. J. Zinn-Justin. "Quantum Field Theory and Critical Phenomena," Oxford University Press, Oxford (1996).

6. J. A. Lipa, D. R. Swanson, J. A. Nissen, T. C. P. Chui, U. E. Israelsson, Phys. Rev. Lett. 76:944 (1996).

7. G. A. Baker Jr., B. G. Nickel, D. I. Meiron, Phys. Rev. B 17:1365 (1978).

8. J. C. Le Guillou, J. Zinn-Justin, Phys. Rev. B 21:3976 (1980).

9. C. Bagnuls, C. Bervillier, Phys. Rev. B 32:7209 (1985).

10. C. Bagnuls, C. Bervillier, D. I. Meiron, B. G. Nickel, Phys. Rev. B 35:3585 (1987).

11. R. Schloms, V. Dohm, Europhys. Lett. 3:413 (1987).

12. R. Schloms, V. Dohm, Nucl. Phys. B 328:639 (1989).

13. R. Schloms, V. Dohm, Phys. Rev. B 42:6142 (1990).

14. I. A. Vakarchuk, Theor. Math. Phys. (Moscow) 36:122 (1978).

15. B. I. Halperin, T. C. Lubensky, S. Ma, Phys. Rev. Lett. 32:292 (1974).

16. J. Lobb, Phys. Rev. B 36:3930 (1987).

17. S. E. Inderhees, M. B. Salamon, N. Goldenfeld, J. P. Rice, B. G. Pazol, D. M. Ginsberg, J. Z. Liu, G. W. Crabtree, Phys. Rev. Lett. 60:1178 (1988). 
18. M. B. Salamon, S. E. Inderhees, J. P. Rice, B. G. Pazol, D. M. Ginsberg, N. Goldenfeld, Phys. Rev. B 38:885 (1988).

19. S. E. Inderhees, M. B. Salamon, J. P. Rice, D. M. Ginsberg, Phys. Rev. Lett. $66: 232$ (1991).

20. S. Regan, A. J. Lowe, M. A. Howson, J. Phys.: Condens. Matter 3:9245 (1991).

21. G. Mozurkewich, M. B. Salamon, Phys. Rev. B 46:11914 (1992).

22. M. B. Salamon, J. Shi, N. Overend, M. A. Howson, Phys. Rev. B 47: 5520 (1993).

23. A. Junod, E. Bonjour, R. Calemczuk, J. Y. Henry, J. Muller, G. Triscone, J. C. Vallier, Physica C 211:304 (1993).

24. N. Overend, M. A. Howson, I. D. Lawrie, Phys. Rev. Lett. 72:3238 (1994).

25. I. D. Lawrie, Phys. Rev. B 50:9456 (1994).

26. J. H. Chen, T. C. Lubensky, D. R. Nelson, Phys. Rev. B 17:4274 (1978).

27. J. C. Le Guillou, E. Brézin, J. Zinn-Justin, Phys. Rev. D 15:1544 (1977).

28. L. N. Lipatov, Sov. Phys. JETP. 45:216 (1977).

29. E. Brezin, G. Parisi, J. Stat. Phys. 19:269 (1978).

30. S. Kolnberger, R. Folk, Phys. Rev. B 41:4083 (1990)

31. R. Folk, Yu. Holovatch, J. Phys. A: Math. Gen. 29:3409 (1996).

32. R. Folk, Yu. Holovatch, J. Phys. Stud. (Lviv) 1:343 (1997).

33. H. Meyer-Ortmanns, Rev. Mod. Phys. 68:473 (1996)

34. Recall that for the given Landau-Ginsburg parameter (ratio of the penetration depth to the coherence length) $k$ a superconductor with $k<1 / \sqrt{2}$ is called type-I and the one with $k>1 / \sqrt{2}$ is called type-II.

35. K. G. Wilson, M. E. Fisher, Phys. Rev. Lett. 28:240 (1972).

36. A. E. Filippov, A. V. Radievsky, A. S. Zeltser, Phys. Lett. A 192:131 (1994); A. S. Zeltser, A. E. Filippov, Jurn. Exp. Theor. Phys. 106:1117 (1994) (in Russian).

37. A. P. C. Malbouisson, F. S. Nogueira, N. F. Svaiter, Europhys. Lett. 41:547 (1998).

38. S. Coleman, E. Weinberg, Phys. Rev. D 7:1988 (1973).

39. J. S. Kang, Phys. Rev. D 10:3455 (1974).

40. I. D. Lawrie, Nucl. Phys. B 200:1 (1982).

41. S. Hikami, Progr. Theor. Phys. 26:226 (1979).

42. S. W. Lovesey, Z. Physik B Condensed Matter 40:117 (1980)

43. C. Dasgupta, B. I. Halperin, Phys. Rev. Lett. 47:1556 (1981).

44. J. Bartholomew, Phys. Rev. B 28:5378 (1983).

45. N. C. Tonchev, D. I. Uzunov, J. Phys. A 14:521 (1981).

46. D. Boyanovsky, J. L. Cardy, Phys. Rev. B 25:7058 (1982).

47. D. I. Uzunov, E. R. Korutcheva, Y. T. Millev, J. Phys. A 16:247 (1983).

48. C. Athorne, I. D. Lawrie, Nucl. Phys. B 265:551 (1986)

49. G. Busiello, L. De Cesare, D. I. Uzunov, Phys. Rev. B 34:4932 (1986).

50. E. J. Blagoeva, G. Busiello, L. De Cesare, Y. T. Millev, I. Rabuffo, D. I. Uzunov, Phys. Rev. B 42:6124 (1990).

51. G. Busiello, L. De Cesare, Y. T. Millev, I. Rabuffo, D. I. Uzunov, Phys. Rev. B 43:1150 (1991).

52. The expression of the $\zeta$-function for the mass aggrees with C. Ford, I. Jack, D. Jones, Nucl. Phys. B387:373 (1992).

53. I. D. Lawrie, C. Athorne, J. Phys. A: Math. Gen. 16:L587 (1983).

54. B. Bergerhoff, F. Freire, D. F. Litim, S. Lola, C. Wetterich, Phys. Rev. B 53:5734 (1996).

55. N. Tetradis, C. Wetterich, Nucl. Phys. B 422:541 (1994).

56. M. Gräter, C. Wetterich, Phys. Rev. Lett. 75:378 (1995).

57. P. Arnold, L. G. Yaffe, Phys. Rev. D 49:3003 (1994); P. Arnold, L. G. Yaffe, Phys. Rev. D 55:1114 (1997) (erratum).

58. H. Kleinert, Lett. Nouvo Cimento 35:405 (1982) (we are thankful to Prof. H. Kleinert for attracting our attention to this reference).

59. M. Kiometzis, H. Kleinert, A. M. J. Schakel, Phys. Rev. Lett. 73:1975 (1994), and Fortschr. Phys. 43:697 (1995).

60. A. J. Bray, Phys. Rev. Lett. 32:1413 (1974).

61. L. Radzihovsky, Europhys. Lett. 29:227 (1995).

62. I. F. Herbut, Z. Tes̆anović, Phys. Rev. Lett. 76:4588 (1996).

63. I. F. Herbut, J. Phys. A 30:423 (1997).

64. In fact an extensive two loop calculation have been already performed earlier by M. Machacek and M. Vaughn, Nucl. Phys. B222:83 (1983); B236:221 (1984); B249:70 (1985).

65. P. Olsson, S. Teitel, Phys. Rev. Lett. 80:1964 (1998). 
66. $\quad$ see e.g. X. Wen, Y. Wu, Phys. Rev. Lett. 70:1501 (1993) and L. Pryadko, S. Zhang, Phys. Rev. Lett. $73: 3282$ (1994).

67. K. K. Nanda, B. Kalta, Phys. Rev. B 57:123 (1998).

68. P. G. de Gennes, Solid State Commun. 10:753 (1972).

69. B. I. Halperin, T. C. Lubensky, Solid State Commun. 14:997 (1974).

70. T. C. Lubensky, J.-H. Chen, Phys. Rev. B 17:366 (1978).

71. G. B. Kasting, K. J. Lushington, C. W. Garland, Phys. Rev. B 22:321 (1980).

72. M. A. Anisimov, P. E. Cladis, E. E. Gorodetskii, D. A. Huse, V. E. Podneks, V. G. Taratuta, W. van Saarloos, V. P. Voronov, Phys. Rev. A 41:6749 (1990).

73. For the most updated comprehensive review of the experimental data on effective critical exponents governing nematic-smectic-A phase transitions see: C. W. Garland, G. Nounesis, Phys. Rev. E 49:2964 (1994).

74. G. t'Hooft, M. Veltman, Nucl. Phys. B 44:189 (1972).

75. The first index is the number of $\Psi$ fields, the second index is the number of $A$ fields.

76. As an example for a determination of the critical exponents values for models with complicated symmetry by applying the resummation technique in different RG schemes see ${ }^{77,78,79,80,81}$; N. A. Shpot, Phys.Lett. A 142:474 (1989); S. A. Antonenko, A. I. Sokolov, Phys. Rev. B 49:15901 (1994); C. von Ferber, Yu. Holovatch, Europhys. Lett. 39:31 (1997), Phys. Rev. E 56:6370 (1997); H. Kleinert, S. Thoms, V. Schulte-Frohlinde, preprint (1997).

77. G. Jug, Phys. Rev. B 27:609 (1983).

78. I. O. Mayer, A. I. Sokolov, B. N. Shalaev, Ferroelectrics 95:93 (1989).

79. I. O. Mayer, J. Phys. A 22:2815 (1989).

80. Yu. Holovatch, M. Shpot, J. Stat. Phys. 66:867 (1989); Yu. Holovatch, T. Yavors'kii, (1998) submitted to J. Stat. Phys.

81. H. K. Janssen, K. Oerding, E. Sengespeick, J. Phys. A: Math. Gen. 28:6073 (1995).

82. G. Grinstein, A. Luther, Phys. Rev. B 13:1329 (1976)

83. G.H. Hardy. "Divergent Series," Oxford University, Oxford (1948).

84. Only when one of the couplings is equal to zero one does obtain a series which is proven to be asymptotic.

85. P. W. Mitchell, R. A. Cowley, H. Yoshizawa, P. Böni, Y. J. Uemura, R. J. Birgeneau, Phys. Rev. B 34:4719 (1986).

86. T. R. Thurston, C. J. Peters, R. J. Birgeneau, P. M. Horn, Phys. Rev. B 37:9559 (1988).

87. J.-S. Wang, M. Wöhlert, H. Mühlenbein, D. Chowdhury, Physica A 166:173 (1990).

88. J.-S. Wang, W. Selke, Vl. S. Dotsenko, V. B. Andreichenko, Europhys. Lett. 11:301 (1994); A. L. Talapov, L. N. Shchur, Europhys. Lett. 27:193 (1994).

89. T. Holey, M. Fähnle, Phys. Rev. B 41:11709 (1990).

90. The results of the resummation appear to be quite insensitive to the choice of $p$.

91. J. S. R. Chisholm, Math. Comp. 27:841 (1973).

92. P. J. S. Watson, J. Phys. A 7:L167 (1974).

93. G. A. Baker Jr., P. Graves-Morris. "Padé approximants," Addison-Wesley Publ. Co., Reading, Mass. (1981).

94. G. Parisi, in: "Proceedings of the 1973 Cargrése Summer School," unpublished. G. Parisi, J. Stat. Phys. 23:49 (1980).

95. H. Kleinert, V. Schulte-Frohlinde, Phys. Lett. B 342:284 (1995).

96. For this model the system of fixed point equations is degenerated at the one-loop level, resulting in particular in the $\sqrt{\varepsilon}$-expansion for the critical exponents: D. E. Khmelnitskii, Zh. Eksp. Theor. Fiz. 68:1960 (1975); T. C. Lubensky, Phys. Rev. B 11:3573 (1975); ${ }^{82}$.

97. It corresponds to a $n=0$ fixed point of the $O(n)$ symmetrical model, described by the de Gennes limit of the self avoiding walk problem.

98. The results are given for the value of the parameter $p=0$ in the Borel-Leroy image.

99. Yu. Holovatch, Preprint, C.E.Saclay, Service de Physique Theorique; S Ph T / 92 - 123); Yu. Holovatch, Int. J. Mod. Phys. A 8:5329 (1993).

100. The last possibility has been chosen by G. Parisi ${ }^{94}$ in order to restore the presence of a stable solution for the fixed point in the two-loop approximation.

101. The series in (37) appears to be an alternating one and this scheme can be applied without any difficulties.

102. M. Abramowitz, A. I. Stegun, (editors) "Handbook of Mathematical Functions with Formulas, Graphs and Mathematical Tables," National Bureau of Standards (1964).

103. In this case the principal value of the integral (29) could be taken, but generally speaking it is preferable to avoid such situations ( $\sec ^{7}$ as well). 
104. We take $\ell_{0}=1$.

105. The value of $\eta$ has been found by the scaling law: $\eta=2-\gamma / \nu$.

106. V. Dohm, Phys. Rev. Lett. 53:1379 (1984).

107. V. Dohm, in: "Application of field theory to statistical mechanics," L. Garrido Ed. p 263, Berlin, Heidelberg, New York, Tokyo, Springer (1985).

108. V. Dohm, Z. Physik Condensed Matter 60:61 (1985).

109. A. Singsaas and G. Ahlers, Phys. Rev. B 30:5103 (1984).

110. J. F. Annett, S. R. Renn, Phys. Rev. B 38:4660 (1988).

111. E. K. Riedel, F. J. Wegner, Phys. Rev. B 9:294 (1974).

112. In fact we have solved the flow equations (57), (58) starting near the unstable fixed points, for the initial value of the flow parameter we have taken $\ell=1$. The use of different initial values $(u(1)$ and $f(1)$ on the seperatrix) would amount to rescale the flow parameter.

113. I. D. Lawrie, Phys. Rev. Lett. 79:131 (1997); see also K. K. Nanda, B. Kalta, Phys. Rev. B $57: 123$ (1998).

114. Because they are double series in two coupling constants we have made use of representing them in the form of resolvent series which has enabled us then to pass to the Padé analysis. 